\title{
FINAL
}

$$
\text { DOE/CH/10539--TI }
$$

\section{Report on \\ Accelerated Life Test of the \\ USDOE OC-OTEC Experimental System \\ Refurbished with Magnetic Bearings \\ for the 3rd Stage Vacuum Compressor}

\author{
Prepared for \\ U.S. Dept. of Energy \\ NREL Area Office 9 \\ DE-AC36-92CH1053ฎ \\ Prepared by \\ Luis A. Vega, Ph.D. \\ Pacific International Center for \\ High Technology Research
}

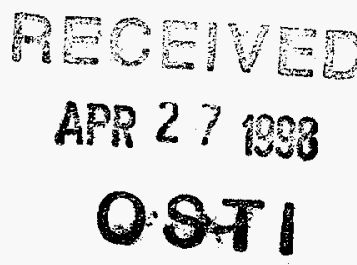

DISTRIBUTION OF THIS DOCUMENT IS UMLMMTED

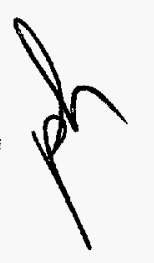

MASTER 


\section{DISCLAIMER}

This report was prepared as an account of work sponsored by an agency of the United States Government. Neither the United States Government nor any agency thereof, nor any of their employees, makes any warranty, express or implied, or assumes any legal liability or responsibility for the accuracy, completeness, or usefulness of any information, apparatus, product, or process disclosed, or represents that its use would not infringe privately owned rights. Reference herein to any specific commercial product, process, or service by trade name, trademark, manufacturer, or otherwise does not necessarily constitute or imply its endorsement, recommendation, or favoring by the United States Government or any agency thereof. The views and opinions of authors expressed herein do not necessarily state or reflect those of the United States Government or any agency thereof. 


\section{DISCLAIMER}

Portions of this document may be illegible electronic image products. Images are produced from the best available original document. 


\section{Executive Summary}

This report documents the accelerated life test performed to evaluate the viability of the magnetic bearing system installed in the stage 3 vacuum pump of the OC-OTEC Experimental Apparatus. PICHTR was directed by DOE to operate the OC-OTEC plant for at least 500 hours over a three month period, with the system operating at $100 \%$ design and above.

It had been previously determined that the maximum achievable operating parameters for the OC-OTEC plant are dictated by the speed and (motor) current limitations of the centrifugal vacuum pumps. As presently configured, without the stage 4 vacuum pump, this results in practical upper limits for system operation given by having the stage 1 and 2 vacuum pumps operate at design speeds and the stage 3 pump at $\approx 94 \%$ of design speed. These limits were observed during the three month test phase reported here.

The plant was operated for 535 hours during the period September-November 1996. The time history records required to evaluate the performance of the system and its subsystems were obtained and stored for on-line and future analysis, as is done whenever the plant is operated. Data records obtained since the initiation of operations in 1993 were previously used to confirm the predicted performance of the experimental OC-OTEC Apparatus. For the purpose of this report, one representative set of records is used to estimate and document the performance of the vacuum compressors subsystem as well as the OC-OTEC system performance during the accelerated life test. This is followed by the discussion of the life tests (time-to-failure) of the vacuum compressors subsystem.

It is concluded that units with magnetic bearings will provide the required thousands of hours of operation. The power consumption estimated for a vacuum compressors subsystem, including a new stage 4 with magnetic bearings, is estimated at $35 \mathrm{~kW}$ under design conditions, meeting the DOE goal of $\leq 40 \mathrm{~kW}$. It is recommended that high speed centrifugal pumps with magnetic bearings be used in future OC-OTEC systems to achieve extended life cycles, relatively low power consumption and, therefore, optimum net power. 


\section{Table of Contents}

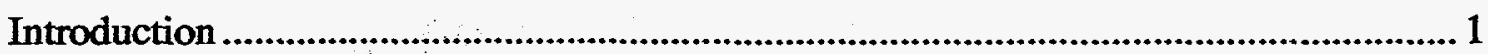

Section I:

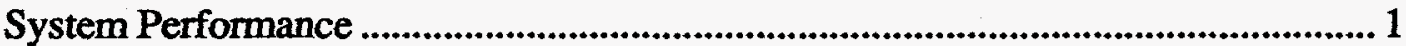

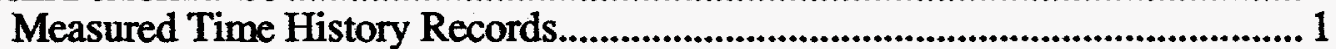

Evaporator Subsystem Performance Parameters .................................................... 3

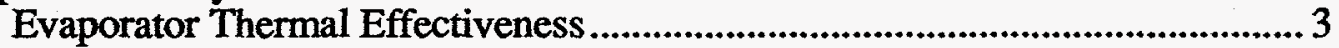

Steam Generation Rate...................................................................... 4

Turbine-Diffuser-Generator Subsystem Performance Parameters ........................... 4

Turbine Isentropic Power ..................................................................................... 4

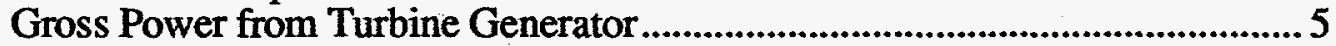

Turbine-Generator System Efficiency …................................................. 5

Direct Contact Condenser Subsystem Performance Parameters ............................. 6

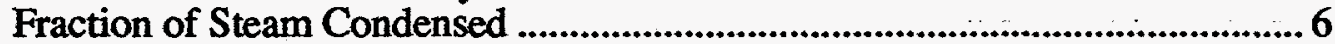

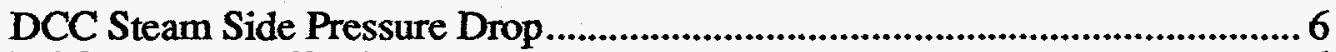

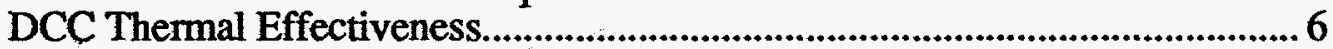

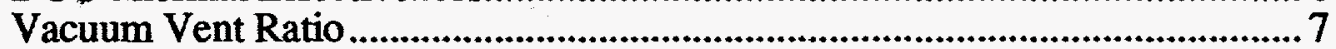

Vacuum Compressor Subsystem Performance Parameters ...................................... 8

Section II:

Vacuum Pumps Life Cycle Data........................................................................... 9

Third Stage Vacuum Compressor Refurbishment .............................................. 10

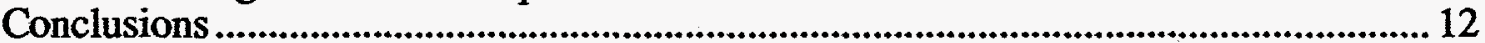

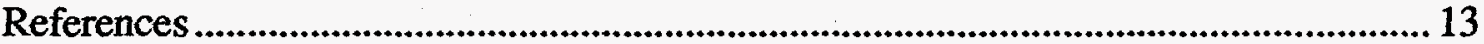

\section{List of Tables}

Table 1A. Stage 1A Centrifugal Compressor .............................................. 14,15

Table 1B. Stage 1B Centrifugal Compressor .................................................... 16,17

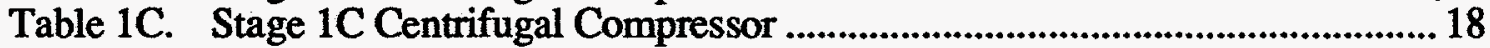

Table 2. Stage 2 Centrifugal Compressor .......................................................... 19

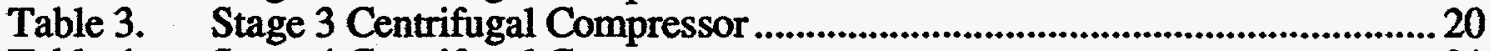

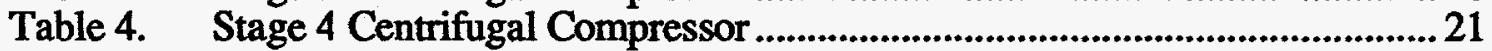

\section{List of Figures}

Figure 1. Evaporator Instrumentation Schematic..............................................22

Figure 2. Turbine-Generator Instrumentation Schematic ..................................... 22

Figure 3. Direct-Contact Condenser Instrumentation Schematic.................................. 23

Figure 4. Vacuum Compressor Subsystem Instrumentation .................................. 23

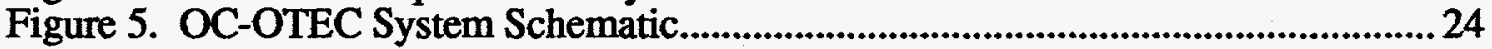

\section{Appendices}

Appendix A Representative Time History Records

Appendix B Uncertainty of Derived Parameters (Power Production Mode) 


\section{Introduction}

This report documents the accelerated life test (time-to-failure) performed, at the request of DOE, to evaluate the viability of the magnetic bearing system installed in the stage 3 vacuum pump. To this effect the plant was successfully operated for over $\mathbf{5 0 0}$ hours during the period September-November 1996. The first part of this report discusses system performance by deriving subsystem and system performance parameters from a typical record. This is followed by the discussion of the life tests. The instrumentation used to estimate the performance parameters given here is depicted in Figures 1 through 5. The third stage pump was operated for 535 hours without incident. It is concluded that magnetic bearings are the preferable choice for the OC-OTEC centrifugal vacuum pumps.

\section{Section I: System Performance}

The OC-OTEC Experimental Apparatus can be considered as encompassing two parts that are tested simultaneously:

(1)Subsystem Tests. Subsystem Tests, in the power production mode, include the performance measurements on (a) spout evaporator (Figure 1), (b) turbine-generator (Figure 2), (c) direct-contact condenser (Figure 3), and (d) vacuum compression system (Figure 4).

(2)System Tests. The system tests focus on the power production of the OC-OTEC system. The system schematic is given in Figure 5.

\section{Measured Time History Records}

The following is a list of all parameters recorded during the accelerated life cycle test:

Warm Seawater Supply Flow Rate

FE-10

Cold Seawater Supply Flow Rate

FE-20

Exhaust Mass Flow Meter (exit of V.C.)

FE-60

Turbine Inlet Total Steam Pressure

PT-32

Diffuser Outlet Steam Pressure

PT-33

st Stage V.C. Inlet Pressure

PT-105

2nd Stage V.C. Inlet Pressure

PT-104

3rd Stage V.C. Inlet Pressure

PT-103

4th Stage V.C. Inlet Pressure

PT-102

5th Stage V.C. Inlet Pressure

PT-101

Turbine Pressure Drop

DPT-31

Diffuser Pressure Recovery

DPT-32

Co-Current (1st Stage) DCC Pressure Drop

DPT-33 


$\begin{array}{ll}\text { Counter-Current (2nd Stage) DCC Pressure Drop } & \text { DPT-34 } \\ \text { Warm Seawater Supply Temperature } & \text { TE-10 } \\ \text { Warm Seawater Discharge Temperature } & \text { TE-12 } \\ \text { Cold Seawater Supply Temperature } & \text { TE-20 } \\ \text { Cold Seawater Discharge Temperature } & \text { TE-22 } \\ \text { Cold Water Temperature Out of DCC 1st Stage } & \text { TE-37,30 \&32 } \\ \text { Cold Water Temperature Out of DCC 2nd Stage } & \text { TE-31 } \\ \text { Turbine Rotational Speed } & \\ \text { Generator Power Output } & \text { SI-41 } \\ \text { Essential Loads Power Consumption } & \text { WT-40 } \\ \text { Non-Essential Loads Power Consumption } & \text { WT-42 } \\ \text { Net Power : (Generator-Essentials) } & \text { WT-43 }\end{array}$

A tabulation of representative time history records, typical of November conditions at the site, is given in Appendix A. The average of these records are used in this report to estimate performance parameters. The uncertainty of derived parameters is discussed in Appendix B. Time history records are routinely recorded for future use and on-line display and analysis. The nominal sampling frequency is set at $1 \mathrm{~Hz}$ (sample/second). The record length and average interval for display purposed is specified by the operator. However, one-hour averages of all measured and derived parameters are routinely displayed along with the record standard deviation, and maximum and minimum values. A minimum of one hour record length is required for every subsystem test.

The records given in Appendix A give the average value of the control parameters resulting in the indicated net power output. The gross power as well as the power consumption are also given. The average control parameters for the 60 minutes long record considered here are :

- Warm water $8419 \mathrm{gpm}$ at $26.33^{\circ} \mathrm{C}$

- Cold water into DCC $5400 \mathrm{gpm}$ at $5.67^{\circ} \mathrm{C}$

- Inlet pressure into Vacuum Pumps $1293 \mathrm{~Pa}$

resulting in

- net power output of $54.8 \mathrm{~kW}$ 


\section{Evaporator Subsystem Performance Parameters}

The performance of the evaporator subsystem is quantified by estimating the value of the thermal effectiveness $\left(\varepsilon_{\text {evap }}\right)$ and steam generation rate $\left(m_{\mathrm{stm}}\right)$.

\section{Evaporator Thermal Effectiveness}

Thermal effectiveness or the evaporator, $\varepsilon_{\text {evap, }}$, is defined as

$$
\varepsilon_{\text {evap }}=\left(T_{w w i}-T_{w w o}\right) /\left(T_{w w i}-T_{\text {satsw }}\right)
$$

where

$$
\begin{aligned}
& \mathrm{T}_{\mathrm{wwi}}=\text { warm-water inlet temperature }\left({ }^{\circ} \mathrm{C}\right) \text {, } \\
& \text { measured by TE-10 (see Figure 1) } \\
& \mathrm{T}_{\text {wwo }}=\text { warm-water outlet (discharge) temperature }\left({ }^{\circ} \mathrm{C}\right. \text { ), } \\
& \text { measured by } \mathrm{TE}-12 \\
& \mathrm{~T}_{\text {satsw }}=\mathrm{T}_{\text {satfw }}+0.31
\end{aligned}
$$

for the range of evaporator pressures measured.

The effectiveness of the evaporator subsystem has been previously estimated to be in the range of 0.80 to 0.95 (e.g., see References). This was determined with a pressure transducer installed in the evaporator chamber that was not available at the time of the life cycle test requested by DOE (September-November 1996). The evaporator instrumentation schematic corresponding to the life-cycle test reported here is given in Figure 1. The value corresponding to the parameters given in Appendix A is 0.8. This is considered to be a lower limit because the pressure measurement was performed downstream of the mist eliminator. The pressure in the evaporator chamber should be approximately $10 \mathrm{~Pa}$ higher than PT-32. 


\section{Steam Generation Rate}

Since the evaporator outlet seawater flow is approximately $99.5 \%$ of the inlet flow, standard flow measurement devices are not accurate enough to determine steam flow from the seawater flow difference. Neither is it feasible to measure the steam flow directly since gas flow-meters are not reliable at the low steam density encountered under open-cycle OTEC conditions.

It was determined that the most accurate method of determining steam generation rate out of the evaporator is balancing the heat absorbed from the seawater with the heat of evaporation of steam:

$$
m_{\text {stm }}=m_{w w i} c_{p s w}\left(T_{w w i}-T_{w w o}\right) / h_{f g}
$$

where

$$
\begin{aligned}
& c_{p s w}=\text { specific heat of seawater }\left(\mathrm{kJ} / \mathrm{kg}-{ }^{\circ} \mathrm{C}\right) \\
& \mathrm{h}_{\mathrm{fg}}=\text { heat of evaporation of steam }(\mathrm{kJ} / \mathrm{kg})
\end{aligned}
$$

the other parameters are defined above.

For the evaporator pressure and temperature and the warm water flow rate and temperatures given in Appendix A, the specific heat of seawater is $4 \mathrm{~kJ} / \mathrm{kg}-{ }^{\circ} \mathrm{C}$; the heat of evaporation of steam $2,451.6 \mathrm{~kJ} / \mathrm{kg}$ resulting in an estimate of $3.41 \mathrm{~kg} / \mathrm{s}$ for the steam generation rate.

\section{Turbine-Diffuser-Generator Subsystem Performance Parameters}

The performance of the turbine subsystem is quantified by measuring the power produced from the generator $\mathrm{PW}_{\mathrm{TG}}$, and the turbine-diffuser exit pressure (i.e., back pressure). These values are $191.6 \mathrm{~kW}$ (WT-40) and $1362 \mathrm{~Pa}$ (PT-33) respectively from the records under discussion. The turbine-generator instrumentation schematic corresponding to the life-cycle test reported here is given in Figure 2.

Turbine Isentropic Power: Isentropic power from the steam turbine is computed based on pressure measurements at the turbine inlet and outlet, and steam flow given above.

For the records given in Appendix A;

$$
\begin{aligned}
& h_{1}=h_{g 1} \text { (enthalpy of dry saturated steam at turbine inlet pressure } \mathrm{kJ} / \mathrm{kg} \text { ) } \\
& h_{1}=2540.3 \mathrm{~kJ} / \mathrm{kg}
\end{aligned}
$$




$$
\begin{aligned}
& s_{1}=s_{\mathrm{g} 1} \text { (entropy of dry saturated steam at turbine inlet pressure } \mathrm{kJ} / \mathrm{kg} /{ }^{\circ} \mathrm{C} \text { ) } \\
& \mathrm{s}_{1}=\mathrm{s}_{\mathrm{g} 1}=8.6426 \mathrm{~kJ} / \mathrm{kg} /{ }^{\circ} \mathrm{C} \\
& \mathrm{x}_{2}=\left(\mathrm{s}_{2}-\mathrm{s}_{\mathrm{f} 2}\right) / \mathrm{s}_{\mathrm{fg} 2} \quad \text { (quality of steam at turbine outlet) }
\end{aligned}
$$

where subscripts 1 and 2 indicate conditions at the inlet and outlet of the turbine-diffuser subsystem. Under isentropic expansion $\left(s_{2}=s_{1}\right)$ at the diffuser outlet the quality and enthalpy of steam are:

$$
\begin{aligned}
& X_{\text {2ise }}=\left(s_{1}-s_{f 2}\right) / s_{f g 2} \\
& h_{2 \text { ise }}=h_{f 2}+x_{2 \text { ise }} \times h_{f g 2}
\end{aligned}
$$

from the ASME steam tables:

$$
\begin{aligned}
& \mathrm{s}_{\mathrm{f} 2}=0.1740 \mathrm{~kJ} / \mathrm{kg} /{ }^{\circ} \mathrm{C} \\
& \mathrm{s}_{\mathrm{fg} 2}=8.6902 \mathrm{~kJ} / \mathrm{kg} /{ }^{\circ} \mathrm{C} \\
& \mathrm{h}_{\mathrm{f} 2}=48.53 \mathrm{~kJ} / \mathrm{kg} \\
& \mathrm{h}_{\mathrm{fg} 2}=2474.2 \mathrm{~kJ} / \mathrm{kg} \\
& \text { resulting in } \\
& \mathrm{X}_{2 \text { ise }}=0.9745 \\
& \mathrm{~h}_{\text {2ise }}=2459.7 \mathrm{~kJ} / \mathrm{kg}
\end{aligned}
$$

yielding an isentropic enthalpy drop (or isentropic expansion specific work) of $\Delta \mathrm{h}_{\text {ise }}=\mathrm{h}_{1}-\mathrm{h}_{2 \text { ise }}$

$$
\Delta \mathrm{h}_{\text {ise }}=2540.3-2459.7=80.6 \mathrm{~kJ} / \mathrm{kg}
$$

such that the isentropic power $\left(=m_{\text {stm }} \times \Delta h_{\text {ise }}\right)$ is:

$$
\text { Isentropic power }=3.41 \times 80.6=274.9 \mathrm{~kW}(\mathrm{~kJ} / \mathrm{s})
$$

Gross Power from Turbine-Generator System: The average gross electrical output from the generator is $191.6 \mathrm{~kW}$ (Appendix A).

Turbine-Generator System Efficiency: The ratio of the gross generator output (WT 40) to the isentropic power yields the combined efficiency of the turbine-diffusergenerator system.

$$
\eta_{\mathrm{TG}}=\mathrm{PW}_{\mathrm{TG}} / \text { isentropic power }
$$

Under conditions encountered during this phase of testing the efficiency is estimated at $70 \%(=191.6 / 274.9)$. The efficiency of the turbine can be estimated, by accounting for 
the mechanical losses and generator efficiency quoted at $15 \mathrm{~kW}$ and $90.7 \%$ respectively by the manufacturer, as $82 \%(=(191.6 / 0.907+15) / 274.9)$.

\section{Direct Contact Condenser Subsystem Performance Parameters}

The performance of the direct-contact-condenser (DCC) subsystem is quantified by estimating the value of the overall fraction of steam condensed $(F)$, steam side pressure drop $\left(\Delta \mathrm{PS} S_{\mathrm{dcc}}\right)$, the thermal effectiveness $\left(\varepsilon_{\mathrm{dcc}}\right)$, and the vacuum vent ratio $(\mathrm{V})$. The DCC instrumentation schematic corresponding to the life-cycle test reported here is given in Figure 3.

\section{Fraction of Steam Condensed}

The overall fraction of steam condensed $(F)$ is estimated by equating the heat transferred to the cold seawater in the DCC, $\mathrm{m}_{\mathrm{cwi}} \mathrm{c}_{\mathrm{psw}}\left(\mathrm{T}_{\mathrm{cwo}}-\mathrm{T}_{\mathrm{cwi}}\right)$, to the energy absorbed from the steam entering the DCC, $X \mathrm{~m}_{\mathrm{stm}} \mathrm{h}_{\mathrm{fg}}$; therefore, the fraction of steam condensed in the DCC is given by:

$$
F=\left(m_{c w i} c_{p s w}\left(T_{c w o}-T_{c w i}\right) /\left(X m_{s t m} h_{f g}\right)\right.
$$

For the parameters given in Appendix A the overall fraction of stem condensed is estimated at 0.98 .

\section{Steam Side Pressure Drop}

The vapor pressure loss is important since it has an impact on the available driving potential for condensation and the inlet pressure seen by the vacuum compressor. The average vapor pressure loss for the co-current stage was $16 \mathrm{~Pa}$ as given by DPT-33 in Appendix A, and for the counter-current stage $60 \mathrm{~Pa}$ as given by DPT-34.

\section{Thermal Effectiveness}

The thermal effectiveness is defined as the ratio of the actual heat-transfer rate to the maximum possible heat-transfer rate. This parameter varies from zero to one with a value of one indicating a perfect device, such as an infinitely long counter-current heat exchanger. For a condenser with non-condensable gases in the vapor, the maximum possible heat transfer is attained when the water outlet temperature is equal to the steam inlet temperature. Assuming a constant liquid specific heat and low condensate to coolant flow ratios, the effectiveness can be defined as :

where

$$
\varepsilon_{\mathrm{dcc}}=\left(\mathrm{T}_{\mathrm{cwo}}-\mathrm{T}_{\mathrm{cwi}}\right) /\left(\mathrm{T}_{\mathrm{satsw}}-\mathrm{T}_{\mathrm{cwi}}\right)
$$

$T_{\text {satsw }}=$ seawater temperature $\left({ }^{\circ} \mathrm{C}\right)$ that is in equilibrium with the steam entering each stage. For the expected range of condenser pressure, this temperature is $0.28^{\circ} \mathrm{C}$ higher than the freshwater saturation temperature. 
The saturation temperature is obtained from the ASME Steam Tables for the pressure at the inlet of the $\mathrm{DCC}\left(11.56^{\circ} \mathrm{C}\right.$ for a pressure of $1362 \mathrm{~Pa}$ in Appendix A)
$\mathrm{T}_{\mathrm{cwi}}=\quad$ cold-water inlet (supply) temperature $\left({ }^{\circ} \mathrm{C}\right)$, measured by the temperature element TE-20,
$\mathrm{T}_{\mathrm{cwo}}=\quad$ temperature $\left({ }^{\circ} \mathrm{C}\right)$ of the seawater exiting the condenser stages. TE-22 for the overall effectiveness

The DCC average overall thermal effectiveness for the typical data given in Appendix A is 0.94 .

\section{Vacuum Vent Ratio}

When non-condensable gases are introduced into the system by leaks and by desorption from the process water, a venting system must be used to remove them and maintain the operating pressure. Under these conditions, a quantity of steam must also be exhausted with the non-condensable gas. The vent ratio compares the actual condenser outlet volumetric flow rate to the ideal outlet volumetric flow rate. The ideal (smallest) volumetric flow rate requires both a condenser with zero pressure losses and one in which the steam reaches the minimum possible partial pressure (the saturation pressure at the inlet water temperature). The vent ration can be expressed as:

$$
V=\frac{\left[P_{i, 1}-\Sigma \Delta P-P_{s a t}\left(T_{s 0,2)}\right]\left(T_{c w i, 2}+273.15\right)\right.}{\left[P_{i, 1}-P_{s a t}\left(T_{c w i, 2}\right)\right]\left(T_{s o, 2}+273.15\right)}
$$

where (values given within parenthesis are from Appendix A):

$$
\begin{aligned}
\mathrm{P}_{\mathrm{i}, 1}= & \text { pressure of vapor (steam and non-condensables) entering the co- } \\
& \text { current condenser stage, measured by PT-33 }(=1362 \mathrm{~Pa})
\end{aligned}
$$

$\Sigma \Delta \mathrm{P}=$ total vapor pressure loss, measured by the sum of DPT -33 and DPT-34 ( = 76 Pa)

$\mathrm{T}_{\mathrm{So}, 2}=$ outlet steam temperature from the counter-current stage, estimated to be equal to the seawater inlet temperature

$P_{\text {Sat }}\left(T_{\text {so,2 }}\right)=$ saturation pressure of the steam exiting the counter-current stage with the outlet temperature $T_{\mathbf{s o}, 2}$. It can be calculated from the Steam Tables as $913 \mathrm{~Pa}$ for a temperature of $5.67^{\circ} \mathrm{C}$ [The pressure 
at the exit of the DCC 1286 PA $(=1362-76)$ is that of the gas, leaving the DCC, containing non-condensable in addition to the steam,

$\mathrm{T}_{\mathrm{cwi}, 2}=$ inlet water temperature for the counter-current stage. It is equal to the temperature of the cold water supply $\left(5.67^{\circ} \mathrm{C}\right)$, measured by TE-20

$P_{\text {sat }}\left(T_{c w i, 2}\right)=$ saturation pressure of the steam in equilibrium with the cold water supply to the counter-current stage $\mathrm{T}_{\mathrm{cwi}, 2}\left(=5.67^{\circ} \mathrm{C}\right)$. It is estimated to be $913 \mathrm{~Pa}$ from the Steam Tables.

The vent ratio is less than 1.0 in a practical condenser because of either one or both of the following; (1) $\mathrm{T}_{\mathrm{so}, 2}$ being slightly higher than $\mathrm{T}_{\mathrm{cwi}, 2}$; or, (2) a drop in the total system pressure (vapor + non-condensable) between the inlet and outlet of the overall DCC, i.e., $\Sigma \Delta P \neq 0$. Under the typical conditions given in Appendix $A$ the vent ratio is estimated at $83 \%$.

\section{Vacuum Compressor Subsystem Performance Parameters}

The performance of the compressor subsystem was quantified by determining the overall power consumption of the compressors $\left(\mathrm{PW}_{\mathrm{nc}}\right)$. In the early stages of the operational phase of the project, before the failure of stage 4 in 1994, the overall power consumption was determined (with the watt-hour meter used to estimate the total power consumed by the vacuum pumps) to be no more than $35 \mathrm{~kW}$ under design conditions. Unfortunately without stage 4 it is necessary to operate the plant with an additional (inefficient) positive displacement compressor resulting in a net increase of power consumption of approximately $20 \mathrm{~kW}$ for a total consumption of approximately $55 \mathrm{~kW}$, or $38 \%$ higher than the DOE goal of $\leq 40 \mathrm{~kW}$. However, given that the third stage vacuum pump refurbished with magnetic bearings does not consume more power than those using grease lubricated bearings, the projected power consumption for a system including a new stage 4 with magnetic bearings would be estimated at $35 \mathrm{~kW}$ under design conditions. What was previously missing was the indication of longer life before failure with the high speed centrifugal compressors. The accelerated life test, discussed in the following Section, indicates that centrifugal compressor using magnetic bearings ought to provide a cost effective solution for OC-OTEC systems.

Compressor parameters like pressure ratio, speed and current, for a given measured mass flow rate of noncondensables, are given in Appendix A. The vacuum compressor subsystem instrumentation schematic corresponding to the life-cycle test reported here is given in Figure 4. 


\section{Section II: Vacuum Pumps Life Cycle Data}

A major problem with the design of the OC-OTEC experimental facility was due, in part, to the emphasis on power consumption (equipment performance) over reliability. The vacuum compression pumps deliver the required aerodynamic performance. Designers and manufacturers of high speed spindles know that the high speed centrifugal vacuum pumps should not have been designed with grease lubricated bearings. Although, during the design phase, excessive bearing temperatures were predicted under operational conditions, this was not entered into the bearing design nor were adequate instruments specified to monitor conditions in and around the bearings. A large effort in the field resulted as complete instrument systems were installed in order to analyze new bearing configurations and predict performance.

The vacuum compression train is not a standard commercial item. The first four stages, designed and manufactured by Barber-Nichols, are high speed centrifugal compressors operating at speeds ranging from about $25,800 \mathrm{rpm}$ (1st stage) to $42,800 \mathrm{rpm}$ (4th stage). To avoid the need for a shaft seal, the electric motor operates in the same vacuum environment as the impeller. The fifth and final stage is a helical screw-type positive displacement unit made by Quincy. A more powerful Quincy unit, called the roughing compressor, was originally intended to be used only for initial vacuum pump down, but can now be operated in that mode or connected in parallel with the 5th stage to provide more pumping capacity during normal operation. For various reasons, including the ability to fine tune system performance to minimize power consumption, the five stages are powered by variable frequency drives (VFD) which allow the compressors to be operated over a broad speed range.

The unreliability of the centrifugal compressors has been the greatest operational problem to date. The failure of these units resulted from the inability to properly cool the bearings. The factors which compound to cause this problem were the use of grease lubricated ball bearings, together with high operating temperatures resulting from the motor and bearings operating in the same vacuum environment as the impeller.

Analysis and field testing indicated that the compressor design was deficient by not accounting for the reduction in the internal-diametral-clearance of the bearing races induced by the relatively high temperature differential $\left(\approx 40^{\circ} \mathrm{C}\right)$ between the inner and outer bearing races. One solution for units operating at speeds below $27,000 \mathrm{rpm}$ has been determined to be to exchange the ceramic balls with smaller balls to accommodate the differential thermal expansion of the bearing cases and the use of a grease which has improved high temperature performance. This redesigned bearing system has resulted in our ability to operate the vacuum pumps for approximately 2,000 hours instead of tens of hours, as in the beginning stages of the project. (See Tables $1 \mathrm{~A}, 1 \mathrm{~B}, 1 \mathrm{C}$ and 2 ). In the case of the higher speed units $(>27,000 \mathrm{rpm}$ ) we selected a magnetic bearing system for the third 
stage and an air bearing system for the fourth stage. To date, the pump refurbished with magnetic bearings has been operated for 535 hours without problems. (See Table 3) It is expected that magnetic bearings will provide the required thousands of hours of operation. Unfortunately, the air bearing system installed in stage 4 failed to operate (Table 4).

\section{Third Stage Vacuum Compressor Refurbishment}

The third stage compressor is a $5 \mathrm{~kW}$ integral motor driven centrifugal compressor, vertically mounted, with a maximum operating speed of 38,500 $\mathrm{rpm}$. Five stages of compression are used to attain the required vacuum. Four of these stages use single stage centrifugal compressors. The fifth is a positive displacement compressor. This letter report concentrates on the third stage compressor that was experiencing bearing reliability problems. The original bearing configuration consisted of spring loaded precision angular contact bearings mounted on each end of the motor rotor, with an overhung impeller. The intent was to convert the compressor to magnetic bearings.

As a first step in the design process in applying magnetic bearings, an estimation of the forces on the rotor is required, both in the radial and axial direction. This is not an easy process, since the forces on a rotor are not generally well known. Static, dynamic, and process fluid forces must all be considered. The dynamic forces can originate from a variety of sources including imbalance, external disturbances, or in this case electrical imbalance from the motor. If the forces are known the bearings can be sized such that they have the capacity to hold the shaft rigidly in place with all these forces combined. In the case of this compressor, these forces were not known well enough to accurately be predicted, and therefore, a conservative estimate was used.

Once this estimation of the force requirements was made by PICHTR, the bearing and rotor layout was undertaken by Revolve. A conical bearing design was chosen to eliminate the need for a thrust bearing, and a tie bolt was used to hole the bearing and motor rotors together as an assembly. The impeller was held onto the tie bolt independently. With this layout completed, the rotor-dynamic analysis was initiated.

As a baseline, information on the free-free modes and frequencies was provided by the compressor OEM. Since the majority of the mass on the rotor was due to the motor rotor and the impeller, it was important to get a good approximation of the effective stiffness diameter of each component. A model of the rotor was generated by Revolve, using an inhouse code, and the relative contribution of stiffness and mass of the motor rotor and impeller were adjusted until good correlation existed between the model and the measured modes.

The next step was to model the rotor with magnetic bearings. The initial design used conical bearings of equal capacity. A shoulder on the tie bolt was located between the 
impeller and impeller end bearing. This allowed the bearings and motor to be stacked up together as one assembly, and the impeller mounted independently. Prediction of the bending modes of the shaft was a difficult task, since the rotor essentially consisted of three complex laminated sleeves held in compression with the tie bolt, and an overhung impeller.

In order to get an accurate estimate of each component's contribution to stiffness, an initial set of bearing rotors were built, assembled on the shaft and tested to determine the undamped natural frequencies and mode shapes. The rotor-dynamic model was adjusted to correlate with the measured frequencies, specifically by adjusting the effective stiffness diameter of each laminated section. It must be noted that great care was taken to ensure good shoulder to shoulder fits between each piece and the tic bolt. Otherwise, the effective stiffness diameter of each piece would be reduced and the corresponding overall shaft stiffness reduced.

Once the model adjustments were completed, a full analysis was undertaken incorporating the active magnetic bearing control system effects. Given the speed of rotation and the relatively large size of the impeller to the shaft, gyroscopic effects were significant. Although our ring tests indicated the first bending mode was at the lower end of the operating range (around $530 \mathrm{~Hz}$ ), our model predicted the gyroscopic stiffening effect would raise the bending mode frequency to the high end of the operating range (around $600 \mathrm{~Hz}$ for the forward whirl mode). It also indicated this mode was poorly damped, meaning operation close to the critical would likely not be possible.

The next step was to verify the model predictions by confirming the location of the mode at speed. These tests confirmed that the mode was in fact where we had predicted, and was poorly camped. This mode would have to be raised in order to attain the desired operating speed range.

Analysis of the mode shapes identified that the location on the rotor where changes in geometry would have the greatest impact was on the impeller and bearing. The design of the rotor was modified until the first bending mode was moved sufficiently out of the operating range.

Several changes were made to the bearing rotor to increase its stiffness contribution. The first was to maximize the lamination inner diameters under the bearing and sensor. This allowed reducing the cone angle of the bearing to reduce its axial force by the rotor weight to match the axial capacity of the upper bearing. Finally, the end ring on the rotor was redesigned to maximize the diameter at which contact was made with the tie bolt shoulder.

The rotor was then reassembled and a ring test performed. Unexpectedly, the bending mode frequency actually decreased. The analysis identified a manufacturing problem, resulting from improper compression of the laminations as the cause. 
The bearing rotor was then rebuilt, assembled on the compressor rotor and re-tested. The ring test now showed that the rotor behaved as predicted with these design changes. With the new design change, full speed was attained without exciting the forward whirl mode. Small amplitude swept sine tests were conducted as a final test to locate the critical at speed to confirm the model once more, and verify a reasonable stability margin existed. The tests by the manufacturer were successful, and installed at the OC-OTEC facility. To date, the third stage pump has been operational for 535 hours without incident. (See Table 3) Magnetic bearings are the preferable choice for OC-OTEC centrifugal vacuum pumps.

\section{Conclusions}

As previously reported (see References), the $210 \mathrm{~kW}$ OC-OTEC Experimental Apparatus has provided valuable data and pointed the way for future modifications and improvements in the OC-OTEC process. The data demonstrate that the OTEC process is technically feasible for the production of base load electricity and desalinated water. Data records obtained since the initiation of operations in 1993 have been previously used to confirm the predicted performance of the experimental OC-OTEC Apparatus.

The most annoying problem has been the frequent failures of the grease lubricated bearings of the centrifugal pumps used for the vacuum and exhaust system. In retrospect this was due to a major design oversight. Equipment operating at speeds higher than approximately $27,000 \mathrm{rpm}$ should, in general, not use grease lubricated bearings. Magnetic bearings like the ones described here are the preferable choice.

Other significant lessons, previously reported, leamed (or relearned) and observations from the perspective of an operator of the OTEC experimental facility are:

- Specifications should be written to emphasize the particulars of the job excluding "boiler plate" information.

- Make the facility "user friendly" from the standpoint of troubleshooting, maintenance, repair and modification

- Include technical field support from suppliers of major equipment, but be prepared to solve most problems on your own.

- Select equipment with excess capacity. It is appropriate to optimize design point performance but there will always be off-design operations requiring additional capacity.

- Mechanical equipment specifically designed for OTEC must be instrumented to measure temperatures and pressures in as many locations as possible. For example, measurements performed with sensors installed, in the field, to estimate temperatures around the bearings 
of the high speed centrifugal pumps revealed that they were failing because of two main causes: (i) deterioration of the bearing's lubricant grease due to high temperatures; and/or (ii) differential expansion of the outer and inner rings resulting in squeezing of the ball bearings.

- If equipment has moving parts evaluate the bearing system and ask potential supplier to provide references of successful application of their design before purchase.

- Consider the corrosive saltwater, condensate, and the typically harsh environment of OTEC sites when making design decisions, especially material selection and placement of mechanical and electrical equipment.

- $\underline{\text { Concrete }}$ is an excellent material for the vacuum structures required for OC-OTEC.

- Avoid metal components, but if unavoidable use the hot-dip-galvanized process from a factory with proven quality. We found that the quality control of most suppliers is poor.

- Fresh Water, instead of seawater, should be used as the Coolant for the intercoolers used with the vacuum compressors.

\section{References}

D. E. Evans, Operational Plan for the $210 \mathrm{~kW}$ OC-OTEC Experimental Apparatus, July 1992. Prepared for the National Renewable Energy Laboratory under Contract No. XC-010077-1.

Pacific International Center for High Technology Research, Final Design of the OpenCycle OTEC Net Power Producing Experiment, November 1991. Prepared for the National Renewable Energy Laboratory under Contract No. XC-0-10077-1.

Idem, As-Built Conditions of the $210 \mathrm{~kW}$ OC-OTEC Experimental Apparatus, December 1992. Prepared for the US Department of Energy under Contract No. DE-AC02-92CH10539.

Idem, $210 \mathrm{~kW}$ OC-OTEC Experimental Apparatus: Significant Lessons Learned, July 1994. Prepared for the US Department of Energy under Contract No. DE-AC02-92CH10539.

Idem, $210 \mathrm{~kW}$ OC-OTEC Experimental Apparatus: Operations from January 1994 to July 1995 (DOE, FY 94), October 1995. Prepared for the US Department of Energy under Contract No. DE-AC02-92CH-10539.

L. A. Vega and D. E. Evans, Operation of a Small Open-Cycle OTEC Experimental Facility, in Proceedings of Oceanology International 94, Vol. 5, Brighton, UK, March 1994.

L. A. Vega, The $210 \mathrm{~kW}$ Open Cycle OTEC Experimental Apparatus: Status Report, in Proceedings of Ocean 95 Conference, San Diego, California, October 1995. 


\begin{tabular}{|c|c|c|c|c|c|c|c|}
\hline \multicolumn{8}{|c|}{ CENTRIFUGALCOMPRESSORSSUMMARY } \\
\hline \multirow[t]{3}{*}{ STAGE } & $1 \mathrm{~A}$ & & & & & & \multirow{4}{*}{ MOTOR } \\
\hline & & & & & & & \\
\hline & & Total Run & \multicolumn{2}{|c|}{ Upper Bearing } & \multicolumn{2}{|c|}{ Lower Bearing } & \\
\hline DATE & Remarks & Time & Type & Grease & Type & Grease & \\
\hline \multirow[t]{2}{*}{$7 / 15 / 93$} & Original & $166 \mathrm{hrs}$ & Fafnir Ceramic & Andok & Same as upper & Same & Original \\
\hline & B-N setup & (meter +61) & Chrome Race & 260 & & & \\
\hline \multirow[t]{3}{*}{$9 / 11 / 93$} & Replaced Both Bearings & $197 \mathrm{hrs}$ & Barden Ceramic & Andok & Barden & Andok & \\
\hline & LB falled visual Inspection & & $\mathrm{C}-105 \mathrm{H}$ & 260 & C. $105 \mathrm{H}$ & 260 & \\
\hline & & & Phenolic Race & & Phenolic Race & & \\
\hline \multirow{6}{*}{$10 / 12 / 93$} & Replaced Upper Bearing & 229 hrs & Failed after & & & & \\
\hline & failed visual inspection. & & 32 hours & & & & \\
\hline & & & Barden & Kluber & Unchanged & & \\
\hline & & & $\mathrm{C}-105 \mathrm{H}$ & & & & \\
\hline & & & & & & & \\
\hline & & & & & & & \\
\hline \multirow[t]{4}{*}{$5 / 29 / 94$} & UB temp. increased & $670 \mathrm{hrs}$ & Failed after & & Unchanged & \multicolumn{2}{|c|}{ Stator Moved } \\
\hline & from $165^{\circ} \mathrm{F}$ to $209^{\circ} \mathrm{F}$ & & 441 hours & & & \multicolumn{2}{|c|}{ to $1 C$, failed } \\
\hline & \multicolumn{2}{|c|}{ in 3 hours. Grease discolored. } & & & & \multicolumn{2}{|c|}{ after 700 hrs } \\
\hline & & & & & & \multicolumn{2}{|c|}{ with 1C } \\
\hline \multirow[t]{3}{*}{$6 / 4 / 94$} & \multicolumn{2}{|l|}{ Installed Reballed Bearings } & Reballed & Kluber & Unchanged & \multicolumn{2}{|r|}{ "Other" } \\
\hline & \multirow{2}{*}{\multicolumn{2}{|c|}{$\begin{array}{l}\text { Lubricated by } \\
\text { Precise, Mr. Zenker }\end{array}$}} & BARDEN & NCA-15 & & \multicolumn{2}{|r|}{ Stator } \\
\hline & & & $\mathrm{C}-105 \mathrm{HX} 118$ & & & & \\
\hline \multirow{4}{*}{$\frac{7 / 27 / 94}{\text { to }}$} & & & & & & & \\
\hline & & & & & & & \\
\hline & Removed unit for & & Unchanged & & Unchanged & & \\
\hline & Inspection \& Decided to & & & & & & \\
\hline \multirow[t]{3}{*}{$8 / 3 / 94$} & Rebalance Impeller & & & & & & \\
\hline & \multicolumn{2}{|l|}{ Reinstalled with same Brgs } & & & & & \\
\hline & & & & & & & \\
\hline
\end{tabular}

TABLE 1A. STAGE TA CENTRIFUGAL COMPRESSOR (Continued) 


\begin{tabular}{|c|c|c|c|c|c|c|c|}
\hline & & Total Run & \multicolumn{2}{|c|}{ Upper Bearing } & \multicolumn{2}{|c|}{ Lower Bearing } & MOTOR \\
\hline DATE & Remarks & Time & Type & Grease & Type & Grease & \\
\hline $9 / 13 / 96$ & High Current Stop & $2128 \mathrm{hrs}$ & \multicolumn{2}{|l|}{ Reballed replaced } & \multicolumn{2}{|l|}{ Standard Barden } & \\
\hline & Remove \& Inspect & & after 1458 hrs & & falled after & & \\
\hline & LB failed & & $(2128-670)$ & & 1931 hrs & & \\
\hline & \multicolumn{2}{|l|}{ UB grease was black decided } & & & $(2128-197)$ & & \\
\hline & to replace & & & & & & \\
\hline & & & & & & & \\
\hline & Both Bearings Replaced & & \multicolumn{2}{|l|}{ Barden C-105HJH } & Zenker & Kluber & \\
\hline & for $24 \mathrm{hrs} /$ day test & & Steel Balls/ & & $\mathrm{C}-105 \mathrm{H} \times 118$ & \multicolumn{2}{|l|}{ NCA-15 } \\
\hline & & & Bronze Cage & & & & \\
\hline & & & & & & & \\
\hline \multirow{3}{*}{$12 / 3 / 96$} & Lower Bearing failed after & $2268 \mathrm{hrs}$ & & & & & \\
\hline & \multicolumn{2}{|c|}{140 hrs due to rotor unbalance } & & & & & \\
\hline & (operator error) & & & & & & \\
\hline \multirow{2}{*}{\multicolumn{8}{|c|}{ - FROM JANUARY 1993 TO MAY 1994 WE EXPERIMENTED WITH DIFFERENT BEARING BRANDS AND GREASES; }} \\
\hline & & & & & & & \\
\hline \multirow{2}{*}{\multicolumn{8}{|c|}{ - SINCE JUNE 1994 WE HAVE BEEN USING REBALLED BEARINGS (SMALLER BALLS, MORE CLERANCE); }} \\
\hline & & & & & & & \\
\hline \multicolumn{8}{|c|}{ - UPPER LOCATION WITH STANDARD BEARINGS ONE FAILURE AFTER 441 HOURS; WITH REBALLED BEARINGS 1458 HRS } \\
\hline \multirow{2}{*}{\multicolumn{6}{|c|}{$\begin{array}{l}\text { LOWER LOCATION WITH STANDARD BEARINGS } 1931 \text { HRS TO FAILURE. } \\
\text {. STANDARD/DESIGN BEARINGS: BARDEN C-105H (Ceramic Balls) WITH ANDOK } 260 \text { GREASE. }\end{array}$}} & & \\
\hline & & & & & & & \\
\hline
\end{tabular}




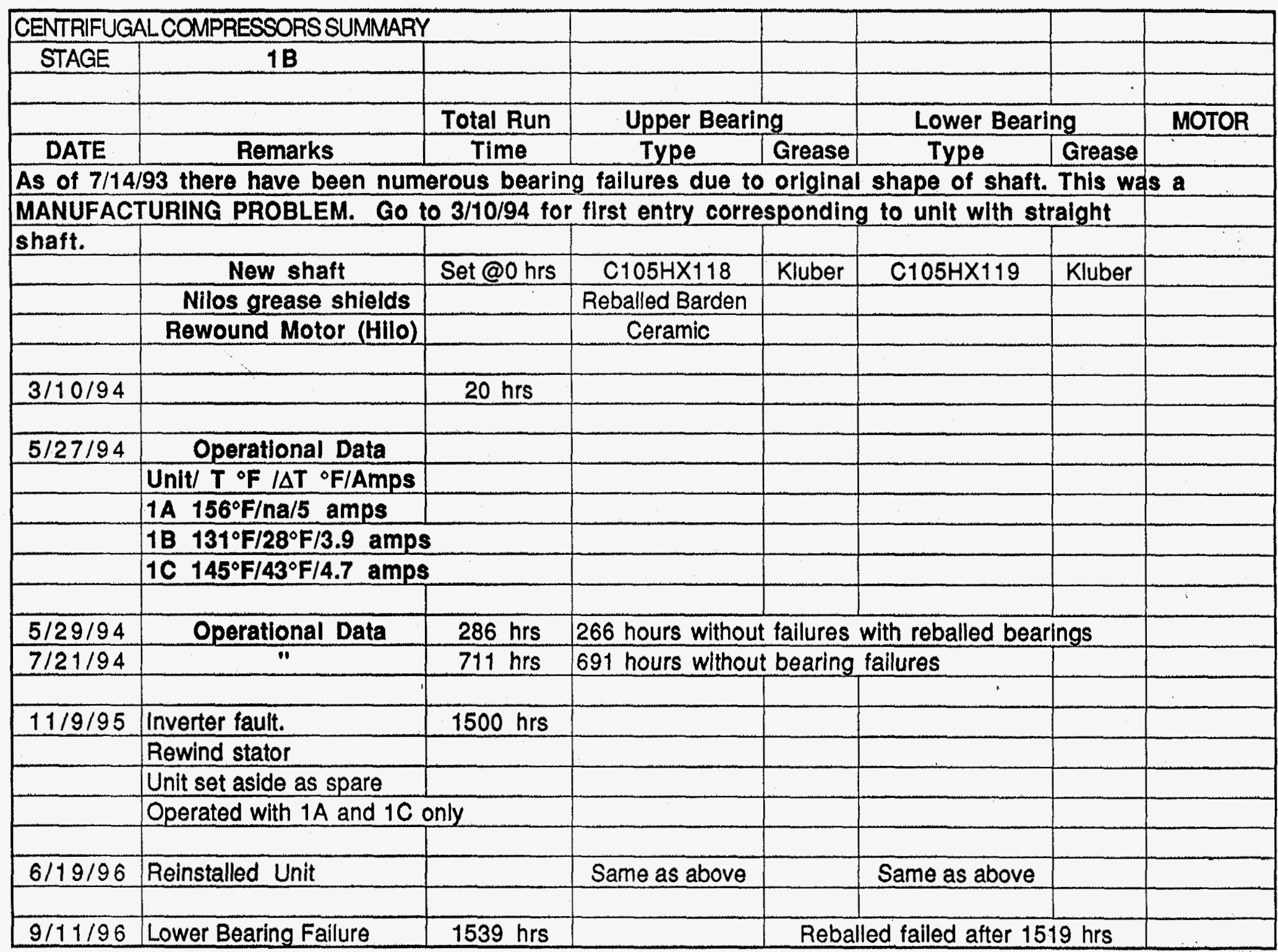

TABLE 1B. STAGE 1B CENTRIFUGAL COMPRESSOR (Continued) 


\begin{tabular}{|c|c|c|c|c|c|c|c|}
\hline & & & & & & \\
\hline & & Total Run & Upper Bearing & Lower Bearing & MOTOR \\
\hline DATE & Remarks & Time & Type & Grease & Type & Grease & \\
\hline $11 / 16 / 96$ & Reinstalled & & 105HJH & Kluber & $105 \mathrm{HJH}$ & Kluber & \\
\hline & & & Steel Balls & & & & \\
\hline & & Bronze Cage & & & & \\
\hline & & & & & & \\
\hline & & & & & & \\
\hline \\
\hline
\end{tabular}




\begin{tabular}{|c|c|c|c|c|c|c|c|}
\hline \multicolumn{8}{|c|}{ CENTRIFUGAL COMPRESSORSSUMMARY } \\
\hline \multirow[t]{2}{*}{ STAGE } & \multicolumn{2}{|l|}{$1 \mathrm{C}$} & & & & & \multirow{3}{*}{ MOTOR } \\
\hline & & & & & & & \\
\hline & & Total Run & \multicolumn{2}{|c|}{ Upper Bearing } & \multicolumn{2}{|c|}{ Lower Bearing } & \\
\hline DATE & Remarks & Time & Type & Grease & Typo & Grease & \\
\hline \multirow[t]{3}{*}{$7 / 14 / 93$} & Original Ceramic Brgs. & $125 \mathrm{hrs}$ & Fafnir Steel & Andok & Fafnir Steel & Andok & Original \\
\hline & Replaced with Steel Balls & & & 260 & & 260 & \\
\hline & to compare with $1 \mathrm{~A}$ & no failures & & & & & \\
\hline & & & & & & & \\
\hline \multirow[t]{2}{*}{$10 / 8 / 93$} & Replaced Upper Bearing & $152 \mathrm{hrs}$ & Barden Steel & Kluber & Unchanged & & \\
\hline & after $27 \mathrm{hrs}$ & & & NBU 15 & & & \\
\hline \multirow[t]{4}{*}{$4 / 5 / 94$} & After 207 hrs. UB grease & 332 hrs & Reballed & Kluber & & & \\
\hline & detiorating & & BARDEN & NCA-15 & & & \\
\hline & \multicolumn{2}{|l|}{ Decided to Replace both with } & C. $105 H \times 118$ & & & & \\
\hline & Reballed Barden/Zenker & & one Nilos Shield & & & & \\
\hline \multirow[t]{4}{*}{$5 / 28 / 94$} & Motor Failure after & $544 \mathrm{hrs}$ & Unchanged & & Unchanged & \multicolumn{2}{|c|}{ Stator from $1 \mathrm{~A}$} \\
\hline & Inverter Fault. & & & & & \multicolumn{2}{|c|}{ moved here } \\
\hline & Replaced with Stator & & & & & \multicolumn{2}{|c|}{ failed after $700 \mathrm{hrs}$} \\
\hline & from $1 \mathrm{~A}$ & & & & & & with $1 \mathrm{C}$ \\
\hline \multicolumn{8}{|l|}{$\cdot$} \\
\hline \multirow[t]{2}{*}{$9 / 24 / 96$} & \multicolumn{2}{|l|}{ VFD Failure after $1853 \mathrm{hrs}$} & Unchanged & & Unchanged & & \\
\hline & Unable to operate Stage 1 & & & & & & \\
\hline \multirow[t]{4}{*}{$11 / 15 / 96$} & High Current & $1990 \mathrm{hrs}$ & & & LB grease gone & & \\
\hline & Manual Trip & & & \multicolumn{3}{|c|}{ Inner race metal-blue color } & \\
\hline & & & \multicolumn{5}{|c|}{ FAILURE after 1865 hrs $(1990-125)$} \\
\hline & & & & & & & \\
\hline \multicolumn{5}{|c|}{ - LOWER LOCATION WITH REBALLED BEARINGS 1865 HRS TO FAILURE. } & & & \\
\hline
\end{tabular}




\begin{tabular}{|c|c|c|c|c|c|c|c|}
\hline \multicolumn{8}{|c|}{ CENTRIFUGAL COMPRESSORS SUMMARY } \\
\hline \multirow[t]{3}{*}{ STAGE } & 2 & & & & & & \multirow{4}{*}{ MOTOR } \\
\hline & & & & & & & \\
\hline & & Total Run & \multicolumn{2}{|c|}{ Upper Bearing } & \multicolumn{2}{|c|}{ Lower Bearing } & \\
\hline DATE & Remarks & Time & Type & Grease & Type & Grease & \\
\hline \multicolumn{8}{|c|}{ Through $9 / 93$ there were several motor fallures (1 original from Colorado plus 3 rewound in Hilo) due to UB Failures } \\
\hline \multicolumn{8}{|c|}{ approximately every $20 \mathrm{hrs}$. The shaft was ruined (bent) and send to Colorado (B-N) for repairs. } \\
\hline \multicolumn{8}{|c|}{ Apparently the shaft was the problem. } \\
\hline \multirow{3}{*}{$9 / 29 / 93$} & 1 nit with Rardan Roaringa & t10 hre & Dardan & Andok & & & \\
\hline & Unit with Barden Bearings & 112 nis & Baraen & Andok & Barden & Andok & \\
\hline & & & C 105H & 260 & C 105H & 260 & \\
\hline \multirow[t]{2}{*}{$5 / 29 / 94$} & Operational Data & 581 hrs & \multicolumn{3}{|c|}{469 hrs $(581-112)$ without bearing failure } & & \\
\hline & No failure & & & & & & \\
\hline & & & & & & & \\
\hline \multirow[t]{5}{*}{$6 / 13 / 94$} & Standard Bearings & $717 \mathrm{hrs}$ & Barden & Krytox & Barden & Krytox & \\
\hline & Falled after 605 hrs & & $\mathrm{C}-105 \mathrm{HX} 118$ & & $\mathrm{C}-105 \mathrm{HX} 118$ & & \\
\hline & Replaced with Reballed & & by us & & & & \\
\hline & \multicolumn{2}{|l|}{ Bearings and Krytox Grease } & & & & & \\
\hline & & & & & & & \\
\hline \multirow[t]{3}{*}{$7 / 21 / 94$} & Bearing Failure after & $793 \mathrm{hrs}$ & Barden & & Barden & & \\
\hline & $76 \mathrm{hrs}$ & & $\mathrm{C}-105 \mathrm{H} \times 118$ & & C.105 HX118 & & \\
\hline & & & by us & & & & \\
\hline \multirow[t]{6}{*}{$6 / 16 / 95$} & Bearing Failure after & 1373 hrs & Reballed & Kluber & Reballed & Kluber & \\
\hline & 580 hrs & & Barden & NCA-15 & Barden & NCA-15 & \\
\hline & This time replaced & & C.105 HX118 & & C-105 HX118 & & \\
\hline & with Reballed Bearings & & Precise/Zenker & & Precise/Zenker & & \\
\hline & Greased by Precise/Zenker & & & & & & \\
\hline & Nylos Shield & & & & & & \\
\hline \multirow{3}{*}{$11 / 24 / 96$} & & & & & & & \\
\hline & Ongoing 1092 hrs. & 2465 hrs & & & & & \\
\hline & with Zenker Bearings & & & & & & \\
\hline
\end{tabular}




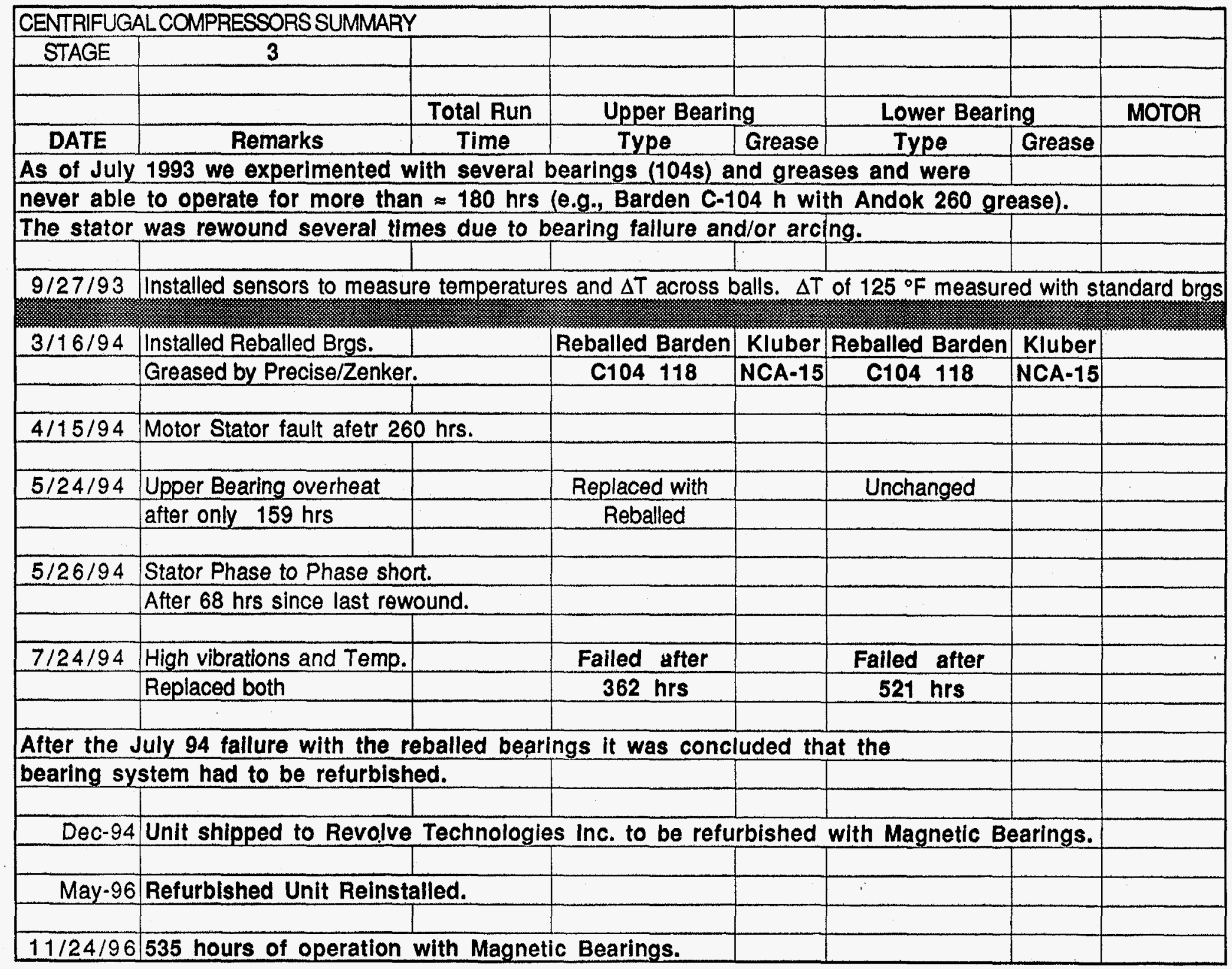




\begin{tabular}{|c|c|c|c|c|c|c|c|}
\hline \multicolumn{8}{|c|}{ CENTRIFUGALCOMPRESSORS SUMMARY } \\
\hline STAGE & 4 & & & & & & \\
\hline & & Total Run & \multicolumn{2}{|c|}{ Upper Bearing } & \multicolumn{2}{|c|}{ Lower Bearing } & MOTOR \\
\hline DATE & Remarks & Time & Type & Grease & Type & Grease & \\
\hline \multicolumn{8}{|c|}{ Through $7 / 93$ there were three bearing fallures and 1 motor failure. } \\
\hline & & & & & & & \\
\hline $7 / 14 / 93$ & Status & & Fafnir Steel & Kluber & Fafnir Ceramic & Kluber & \\
\hline \multirow[t]{3}{*}{$9 / 23 / 93$} & After Failure & & Barden 104 & Andok & Barden 104 & Andok & \\
\hline & Replaced with Barden & & Steel Balls & 260 & Steel Balls & 260 & \\
\hline & Balanced at Field Office & & & & & & \\
\hline & & & & & & & \\
\hline \multirow[t]{3}{*}{$10 / 1 / 93$} & Upper Bearing Failure & & Barden 104 & Texaco & Unchanged & & \\
\hline & After 23 hrs & & Steel Balls & Starflex & & & \\
\hline & & & & & & & \\
\hline \multirow[t]{2}{*}{$10 / 6 / 93$} & UB failure after $4 \mathrm{hrs}$ & & Fafnir & Kluber & Unchanged & & \\
\hline & Grease is not the solution & & Steel & & & & \\
\hline & & & & & & & \\
\hline \multirow[t]{2}{*}{$1 / 18 / 94$} & Lower Bearing Failure & \multicolumn{3}{|c|}{ Shaft bent sent to $B-N$ for repairs } & & & \\
\hline & After 110 hrs & & & & & & \\
\hline & & & & & & & \\
\hline $3 / 11 / 94$ & Installed Reballed Bearings & & Barden 104 & & Barden 104 & & \\
\hline to & But only achieved $\approx 30$ hrs & & Reballed & & Reballed & & \\
\hline $3 / 31 / 94$ & due to poor installation by $u$ & & by us & & by us & & \\
\hline \multirow[t]{4}{*}{$4 / 6 / 94$} & This time Reballed Brgs. & & Reballed & Kluber & Reballed & Kluber & \\
\hline & Greased by Precise/Zenke & & Barden & NCA-15 & Barden & NCA-15 & \\
\hline & & & $C-104$ & & C-104 & & \\
\hline & & & & & & & \\
\hline \multirow[t]{2}{*}{$6 / 7 / 94$} & LB Failure after 260 hrs y & with Reballe & d Bearings & & & & \\
\hline & Concluded that grease be & earings can & not be used fo & the third & d and fourth sta & & \\
\hline Apr-95 & The unit refurbished with & air bearir & s failed. Air & $y$ & $n$ & & \\
\hline
\end{tabular}


OC-OTEC Vacuum Pumps Life Cycle Tests

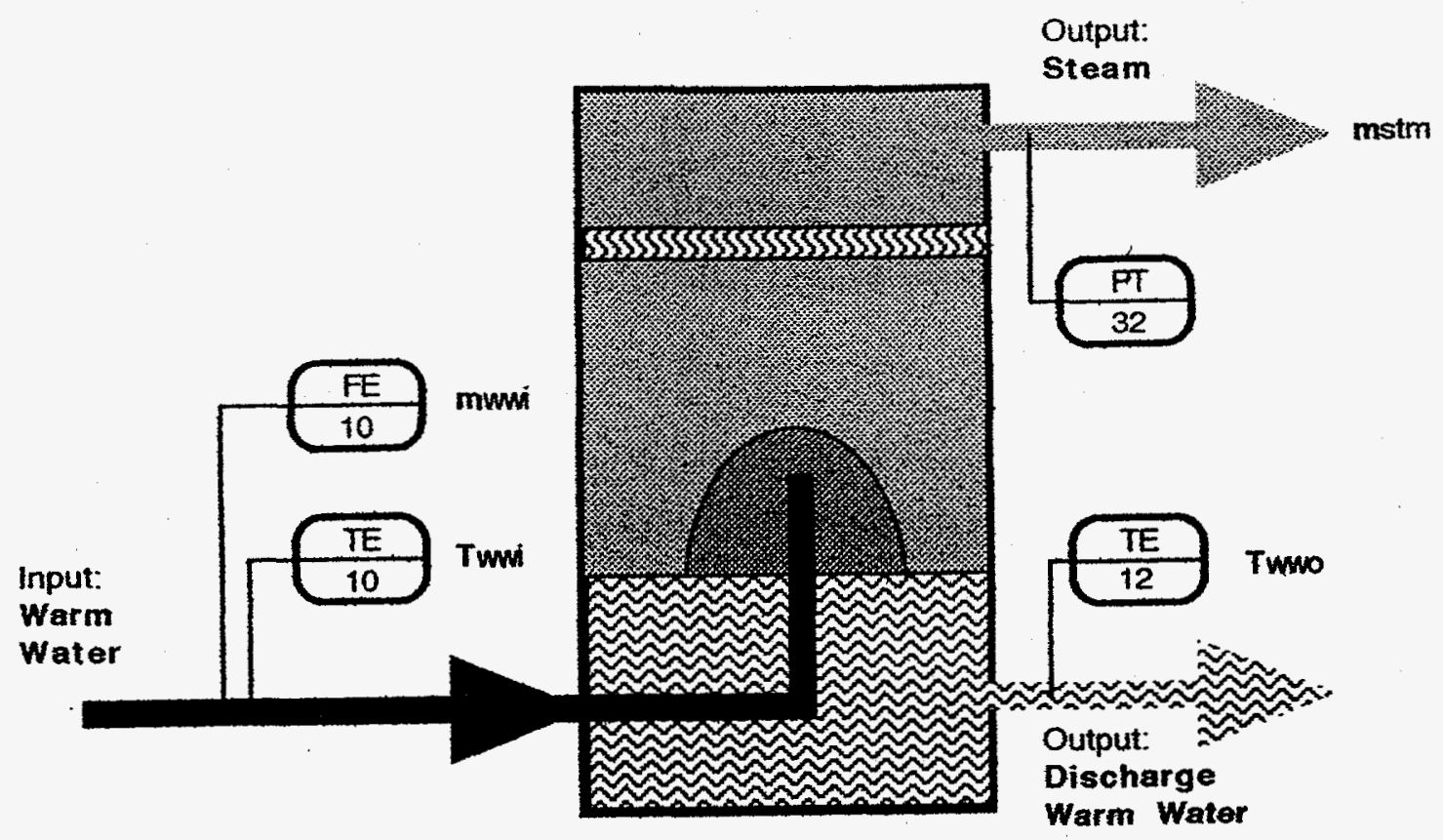

Figure 1.- EVAPORATOR INSTRUMENTATION SCHEMATIC

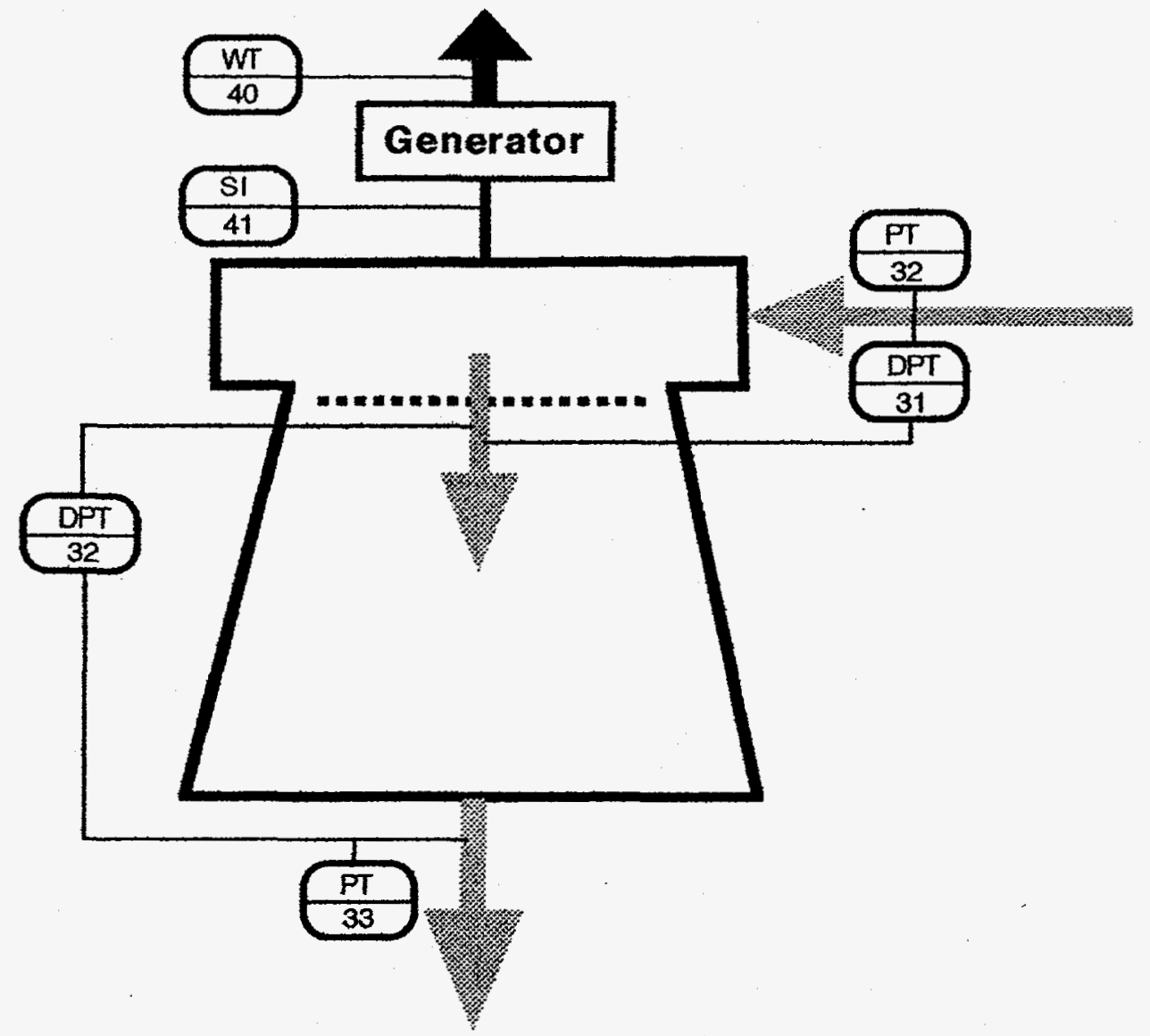

Figure 2.- TURBINE-GENERATOR INSTRUMENTATION SCHEMATIC 


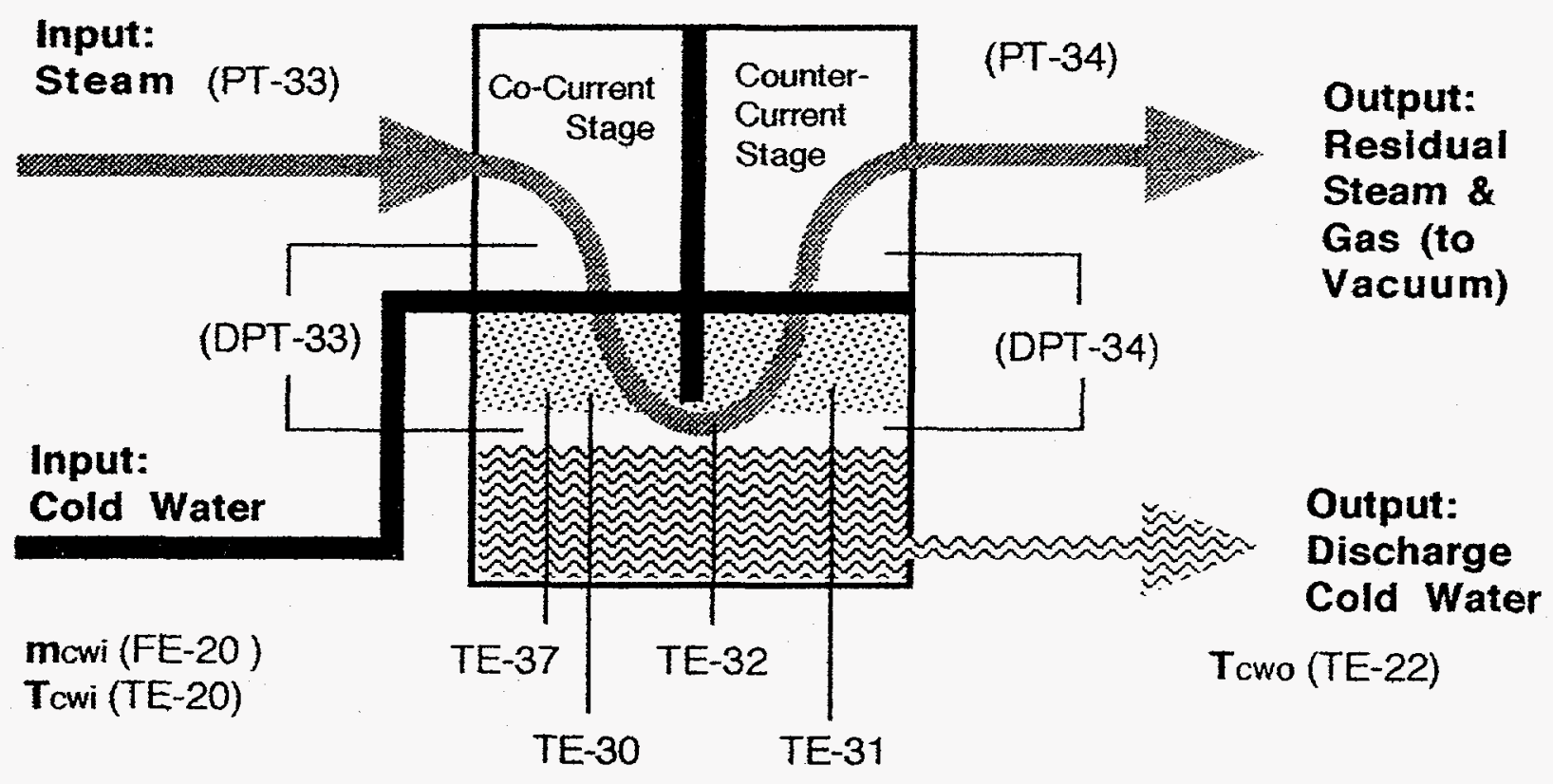

Figure 3.- DIRECT-CONTACT CONDENSER INSTRUMENTATION SCHEMATIC

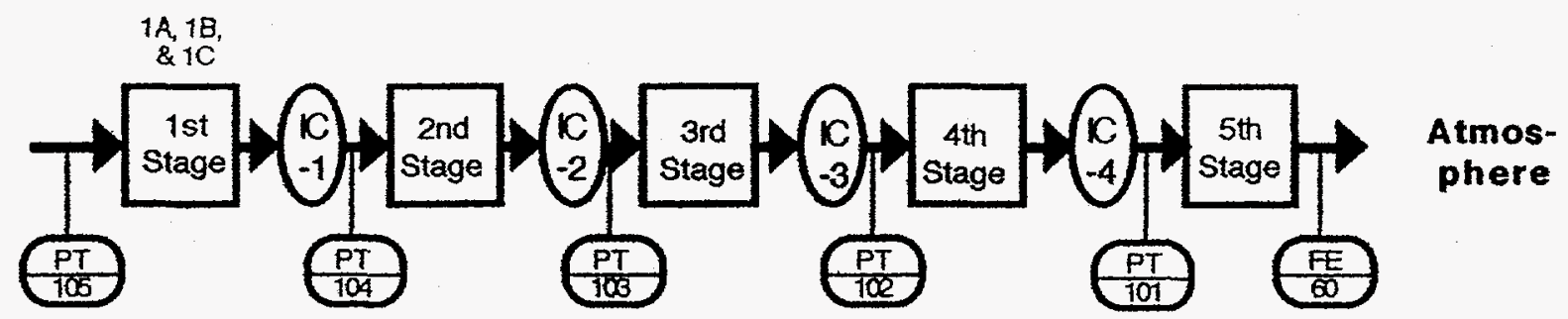

Figure 4.- VACUUM COMPRESSOR SUBSYSTEM INSTRUMENTATION Used during the life-cycle tests

(IC: Intercooler) 


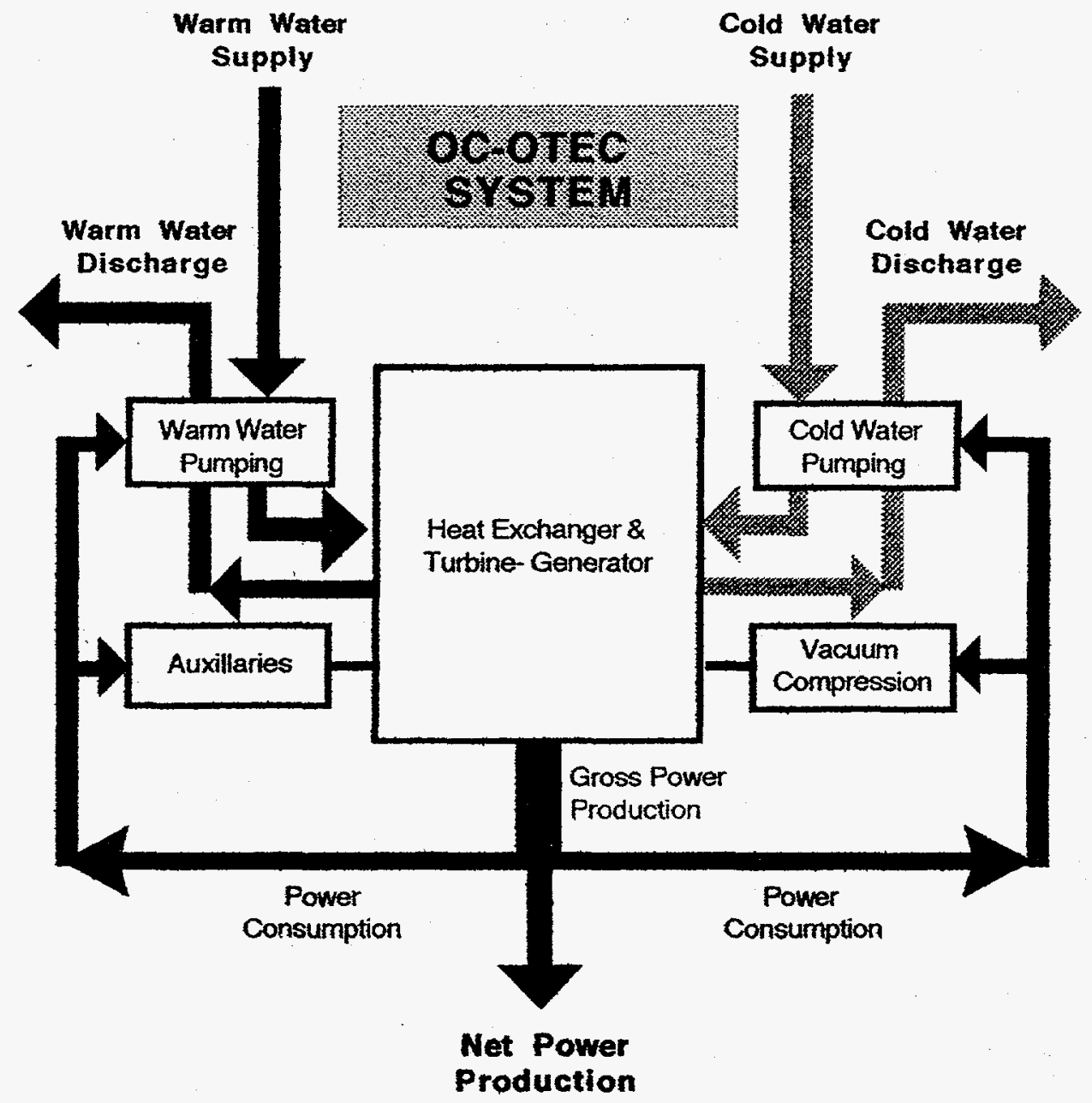

WT-40: Generator Power Output (gross power)

WT-42: Essential Loads (Seawater pumping, vacuum compression and essential auxiliaries)

WT-44: Net Power $=(W T-40)-(W T-42)$

Figure 5.- OC-OTEC SYSTEM SCHEMATIC Used during the life-cycle test 


\section{Appendix A}

\section{Representative Time History Records}

The SI system of units is used here unless otherwise noted. For example, pressures and temperatures are given in $\mathrm{Pa}$ and ${ }^{\circ} \mathrm{C}$ respectively, but water volumetric flowrates in gallons-per-minute (gpm). All power measurements are given in $\mathrm{kW}$ and currents in amperes. Rotational speeds are given in revolutions-per-minute (rpm) and the mass flowrate of noncondensables in $\mathrm{g} / \mathrm{s}$. 
OC-OTEC Vacuum Pumps Life Cycle Tests

Appendix A

\section{Table of Contents}

Control/Output Parameters .....................................................................................3

Evaporator/Turbine Measured Parameters ............................................................4

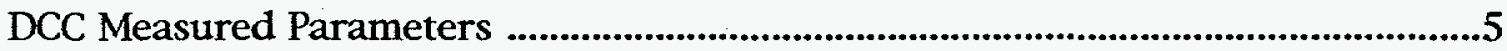

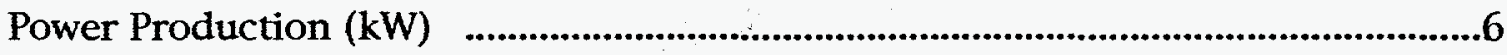

Vacuum Compressor Subsystem Parameters . .............................................................7

Vacuum Compressor Subsystem Parameters .......................................................8 
Vacuum Pump Life Test for the $210 \mathrm{~kW}$ OC-OTEC Experimental Plant

Appendix A

CONTROLOUTPUT PARAMETERS

\begin{tabular}{|c|c|c|c|c|c|c|}
\hline \multicolumn{6}{|c|}{ Time History Record ( 60 minutes long) Typical Nov. Conditions } & \multirow[b]{2}{*}{ PWnet } \\
\hline & Qww & Qcwdoc & Twwin & Towin & Pinve & \\
\hline Time & $\mathrm{gpm}$ & $\mathrm{gpm}$ & $\operatorname{deg} C$ & $\operatorname{deg} C$ & $\mathrm{~Pa}$ & $\mathrm{~kW}$ \\
\hline $10: 20$ & 8345 & 5443 & 26.28 & 5.67 & 1299 & 55.4 \\
\hline $10: 21$ & 8400 & 5460 & 26.28 & 5.55 & 1290 & 55 \\
\hline $10: 22$ & 8416 & 5419 & 26.27 & 5.64 & 1299 & 55.4 \\
\hline 1023 & 8354 & 5425 & 26.29 & 5.66 & 1301 & 56.3 \\
\hline $10: 24$ & 8499 & 5431 & 26.29 & 5.64 & 1300 & 56.4 \\
\hline $10: 25$ & 8502 & 5439 & 26.31 & 5.64 & 1298 & 55.5 \\
\hline $10: 26$ & 8339 & 5442 & 26.29 & 5.65 & 1300 & 54.2 \\
\hline $10: 27$ & 8517 & 5471 & 26.29 & 5.65 & 1300 & 55.4 \\
\hline $10: 28$ & 8374 & 5427 & 26.3 & 5.66 & 1258 & 55.9 \\
\hline $10: 29$ & 3400 & 5474 & 26.31 & $5 . \tilde{5}$ & 1301 & 55.5 \\
\hline $10: 30$ & 8414 & 5466 & 26.31 & 5.5 & 1300 & 55.3 \\
\hline 1031 & 8285 & 5442 & 26.31 & 566 & 1300 & 519 \\
\hline $10: 32$ & 8337 & 5407 & 26.29 & 5.66 & 1302 & 51 \\
\hline $10: 33$ & 8388 & 5432 & 26.3 & 5.66 & 1297 & 56.5 \\
\hline $10: 34$ & 8565 & 5389 & 26.32 & 5.65 & 1300 & 55.6 \\
\hline $10: 35$ & 8456 & 5474 & 26.31 & 5.63 & 1298 & 55.5 \\
\hline $10: 36$ & 8420 & 5314 & 26.3 & 5.62 & 1301 & 55.4 \\
\hline $10: 37$ & 8550 & 5390 & 26.32 & 5.6 & 1297 & 57 \\
\hline 10.38 & 8443 & 5391 & 25.32 & 5.51 & 1290 & 57 \\
\hline 1039 & 8396 & 5302 & 26.35 & 5.62 & 1296 & 56.2 \\
\hline $10: 40$ & 8448 & 5288 & 26.37 & 5.62 & 1293 & 56 \\
\hline $10: 41$ & 8457 & 5363 & 26.37 & 5.62 & 1292 & 56.5 \\
\hline $10: 42$ & 8450 & 5429 & 26.34 & 5.63 & 1293 & 55.7 \\
\hline $10: 43$ & 8322 & 5393 & 26.36 & 5.65 & 1291 & 55.4 \\
\hline $10: 44$ & 8405 & 5354 & 26.36 & 5.66 & 1293 & 55.7 \\
\hline 10.45 & 8559 & 5500 & 26.35 & 5.65 & 1291 & 55.1 \\
\hline $10: 46$ & 8408 & 5492 & 25.35 & 5.66 & 1293 & 56.4 \\
\hline $10: 47$ & 8492 & 5376 & 26.34 & 5.67 & 1293 & 55.4 \\
\hline $10: 48$ & 8328 & 5394 & 26.36 & 5.69 & 1289 & 56.2 \\
\hline $10: 49$ & 8399 & 5434 & 26.36 & 5.68 & 1292 & 54.3 \\
\hline $10: 50$ & 8343 & 5361 & 26.34 & 5.69 & 1290 & 54.8 \\
\hline 1051 & 8461 & 5457 & 26.33 & 5.66 & 1293 & 50.5 \\
\hline 1052 & 8415 & 5395 & 26.33 & 5.67 & 1293 & $50 . \overline{2}$ \\
\hline $10: 53$ & 8501 & 5384 & 26.33 & 5.69 & 1293 & 54 \\
\hline $10: 54$ & 8464 & 5365 & 26.32 & 5.58 & 1288 & 56.4 \\
\hline $10: 55$ & 8533 & 5346 & 26.35 & 5.68 & 1291 & 54 \\
\hline $10: 56$ & 8489 & 5419 & 26.33 & 5.69 & 1292 & 54.7 \\
\hline 10.57 & 8549 & 5425 & 26.34 & 5.69 & 1287 & 55 \\
\hline $10: 58$ & 8370 & 5341 & 26.34 & 5.68 & 1291 & 5.6 \\
\hline $10: 59$ & 8387 & 5276 & 26.34 & 5.68 & 1284 & 56.1 \\
\hline $11: 00$ & 8463 & 5415 & 26.33 & 5.68 & 1286 & 56.1 \\
\hline $11: 01$ & 8378 & 5374 & 26.35 & 5.68 & 1288 & 56.5 \\
\hline 11.02 & 8321 & 5454 & 26.35 & 5.69 & 1289 & 54.8 \\
\hline 11.03 & 8397 & 5379 & 26.38 & 5.67 & 1286 & 55.5 \\
\hline $11: 04$ & 8312 & 5313 & 26.35 & 5.67 & 1289 & 54 \\
\hline $11: 05$ & 8453 & 5368 & 26.37 & 5.66 & 1288 & 55.1 \\
\hline 11:06 & 8436 & 5364 & 26.36 & 5.68 & 1288 & 54.8 \\
\hline $11: 07$ & 8545 & 5363 & 26.37 & 5.68 & 1285 & 55.1 \\
\hline 11.08 & 8375 & 5382 & 26.36 & 5.7 & 1287 & 55 \\
\hline $11: 09$ & 8387 & 5379 & 26.37 & 5.71 & 1287 & 54.6 \\
\hline $11: 10$ & 8359 & 5434 & 26.35 & 5.69 & 1285 & 55.2 \\
\hline $11: 11$ & 8317 & 5395 & 26.36 & 5.69 & 1289 & 50.6 \\
\hline $11: 12$ & 8347 & 5354 & 26.37 & 5.69 & 1287 & 51.2 \\
\hline $11: 13$ & 8423 & 5514 & 26.37 & 5.68 & 1290 & 54.4 \\
\hline $19: 14$ & 8438 & 5442 & 26.35 & 5.69 & 1285 & 55.1 \\
\hline $11: 15$ & 8389 & 5264 & 26.35 & 5.7 & 1287 & 54.6 \\
\hline $11: 16$ & 8377 & 5336 & 26.34 & 5.7 & 1290 & 54.5 \\
\hline $11: 17$ & 8530 & 5387 & 26.31 & 5.71 & 1284 & 54.6 \\
\hline $11: 18$ & 8316 & 5300 & 26.31 & 5.7 & 1291 & 53.6 \\
\hline $11: 19$ & 8398 & 5467 & 26.33 & 5.71 & 1289 & 55 \\
\hline Average & 8419 & 5400 & 26.33 & 5.67 & 1293 & 54.8 \\
\hline Stdev & 72 & 56 & 10.03 & 0.03 & 5 & 1.5 \\
\hline
\end{tabular}


EVAPORATORTTURBINE MEASURED PARAMETERS

\begin{tabular}{|c|c|c|c|c|c|c|c|}
\hline \multicolumn{8}{|c|}{ Time History Record ( 60 minutes long) Typical Nov Conditions } \\
\hline & Tevpin & Tevpout & Pintrb & Pouttrb & DPtrb & Prec. diff & TRBrpm \\
\hline Time & $\mathrm{T} ! 10$ & TL-12 & Pl-32 & P1-33 & PDI-31 & PDI-32 & Si -41 \\
\hline $10: 20$ & 26.28 & 22.51 & 2517 & 1354 & $19 \overline{9}$ & 32 & 1834.4 \\
\hline $10: 21$ & 2628 & 22.48 & 2516 & 1352 & 1199 & 32 & 1834.4 \\
\hline $10: 22$ & 26.27 & 22.48 & 2515 & 1352 & 1197 & 32 & 1834.4 \\
\hline $10: 23$ & 26.29 & 22.5 & 2519 & 1364 & 1201 & 32 & 1834.4 \\
\hline 10.24 & 26.29 & 22.5 & 2517 & 1363 & 1199 & 32 & 1834.4 \\
\hline $10: 25$ & 26.31 & 22.47 & 2515 & 1363 & 1197 & 32 & 1834.4 \\
\hline $10: 26$ & 26.29 & 22.51 & 2516 & 1362 & 1200 & 32 & 1834.4 \\
\hline $10: 27$ & 26.29 & 22.53 & 2517 & 1364 & 1199 & 32 & 1834.4 \\
\hline $10: 28$ & 26.3 & 225 & 2516 & 1361 & 1201 & 33 & 1834.4 \\
\hline $10: 29$ & 26.31 & 225 & 2516 & 1362 & 1200 & 32 & 1834.4 \\
\hline $10: 30$ & 26.31 & 22.49 & 2515 & 1361 & 1200 & 32 & 1834.4 \\
\hline $10: 31$ & 26.31 & 22.48 & 2517 & 1363 & 1200 & 33 & 1834.4 \\
\hline $10: 32$ & 26.29 & 22.49 & 2515 & 1362 & 1199 & 33 & 1834.4 \\
\hline 10.33 & 26.3 & 22.54 & 2520 & 1363 & 1204 & 33 & 1834.4 \\
\hline $10: 34$ & 26.32 & 22.51 & 2518 & 1363 & 1200 & 33 & 1834.4 \\
\hline $10: 35$ & 26.31 & 22.49 & 2514 & 1361 & 1200 & 33 & 1834.4 \\
\hline 10.36 & 26.3 & 2251 & 2515 & 1361 & 1200 & 33 & 1834.4 \\
\hline $10: 37$ & 2632 & 22.48 & 2517 & 1350 & 1206 & 33 & 1834.4 \\
\hline $10: 38$ & 26.32 & 22.48 & 2518 & 1361 & 1204 & 33 & 1834.4 \\
\hline 1039 & 26.35 & 22.46 & 2518 & 1362 & 1202 & 33 & 1834.4 \\
\hline $10: 40$ & 26.37 & 22.46 & 2514 & 1361 & 1200 & 33 & 1834.4 \\
\hline $10: 41$ & 26.37 & 22.48 & 2515 & 1363 & 1199 & 33 & 1834.4 \\
\hline $10: 42$ & 26.34 & 2248 & 2514 & 1363 & 1199 & 32 & 1834.4 \\
\hline $10: 43$ & 26.36 & 2251 & 2516 & 1361 & 1202 & 32 & 1834.4 \\
\hline $10: 44$ & 26.36 & 22.52 & 2511 & 1361 & 1198 & 32 & 1834.4 \\
\hline $10: 45$ & 26.35 & 22.5 & 2510 & 1359 & 1197 & 32 & 1834.4 \\
\hline $10: 46$ & 26.35 & 22.5 & 2512 & 1360 & 1199 & 31 & 1834.4 \\
\hline $10: 47$ & 26.34 & 22.5 & 2510 & 1361 & 1196 & 31 & 1834.4 \\
\hline $10: 48$ & 26.36 & 22.48 & 2510 & 1361 & 1196 & 31 & 18344 \\
\hline $10: 49$ & 26.36 & 22.49 & 2507 & 1362 & 1192 & 31 & 1834.4 \\
\hline 10.50 & 26.34 & 22.48 & 2505 & 1359 & 1194 & 31 & 1834.4 \\
\hline 10.51 & 26.33 & 22.44 & 2508 & 1360 & 1195 & 31 & 1834.4 \\
\hline $10: 52$ & 26.33 & 22.42 & 2505 & 1359 & 1194 & 31 & 1834.4 \\
\hline 10.53 & 26.33 & 22.44 & 2507 & 1360 & 1195 & 31 & 1834.4 \\
\hline $10-54$ & 26.32 & 2247 & 2500 & 1361 & 1195 & 31 & 1834.4 \\
\hline $10: 55$ & 26.35 & 22.47 & 2507 & 1361 & 1194 & 31 & 1834.4 \\
\hline $10: 56$ & 26.33 & 22.47 & 2508 & 1360 & 1196 & 31 & 1834.4 \\
\hline $10: 57$ & 26.34 & 22.48 & 2504 & 1357 & 1195 & 31 & 1834.4 \\
\hline 10.58 & 26.34 & 22.49 & 2505 & 1359 & 1194 & 31 & 1834.4 \\
\hline 10.59 & 26.34 & 22.46 & 2506 & 1359 & 1194 & 31 & 1834.4 \\
\hline $11: 00$ & 2633 & 22.46 & 2507 & 1360 & 1195 & 31 & 1834.4 \\
\hline $11: 01$ & 26.35 & 22.46 & 2508 & 1361 & 1196 & 31 & 1834.4 \\
\hline 11.02 & 26.35 & 22.45 & 2500 & 1362 & 1196 & 31 & 1834.4 \\
\hline 11.03 & 26.38 & 22.49 & 2509 & 1362 & 1195 & 30 & 1834.4 \\
\hline 11.04 & 26.35 & 22.51 & 2508 & 1362 & 1194 & 30 & 1834.4 \\
\hline 11.05 & 26.37 & 225 & 2511 & 1363 & 1197 & 30 & 1834.4 \\
\hline 11.06 & 26.36 & 22.5 & 2510 & 1364 & 1194 & 30 & 1834.4 \\
\hline 11.07 & 26.37 & 22.49 & 2508 & 1363 & 1193 & 30 & 1834.4 \\
\hline 11.08 & 26.36 & 22.48 & 2508 & 1364 & 1192 & 30 & 1834.4 \\
\hline 11.09 & 26.37 & 22.5 & 2508 & 1363 & 1191 & 30 & 1834.4 \\
\hline $11: 10$ & 26.35 & 22.47 & 2508 & 1362 & 1194 & 29 & 1834.4 \\
\hline $11: 11$ & 26.36 & 22.49 & 2511 & 1364 & 1194 & 29 & 1834.4 \\
\hline $11: 12$ & 26.37 & 22.47 & 2512 & 1364 & 1196 & 29 & 1834.4 \\
\hline $11: 13$ & 26.37 & 22.49 & 2508 & 1364 & 1191 & 29 & 1834.4 \\
\hline $11: 14$ & 26.35 & 22.51 & 2510 & 1364 & 1194 & 29 & 1834.4 \\
\hline $11: 15$ & 26.35 & 22.49 & 2511 & 1365 & 1194 & 29 & 1834.4 \\
\hline $11: 16$ & 26.34 & 22.48 & 2500 & 1366 & 1191 & 29 & 1834.4 \\
\hline $11: 17$ & 26.31 & 22.46 & 2513 & 1368 & 1192 & 29 & 1834.4 \\
\hline $11: 18$ & 26.31 & 22.46 & 2507 & 1366 & 1188 & 29 & 1834.4 \\
\hline $11: 19$ & 26.33 & 22.49 & 2512 & 1365 & 1194 & 30 & 1834.4 \\
\hline Average & 26.33 & 22.48 & 2512 & 1362 & 1197 & 31 & 1834.4 \\
\hline Stdev & 0.03 & 0.02 & 4 & 2 & 4 & 1 & 0 \\
\hline
\end{tabular}


Vacuum Pump Life Test for the $210 \mathrm{~kW}$ OC-OTEC Experimental Plant

Appendix A

DCC MEASURED PARAMETERS

\begin{tabular}{|c|c|c|c|c|c|c|c|c|c|}
\hline \multicolumn{5}{|c|}{ Time History Record Typical Nov. Conditions } & \multirow[b]{2}{*}{ DP2nddoc } & \multirow{2}{*}{$\begin{array}{l}\text { Tiststg } \\
\text { outer }\end{array}$} & \multirow{2}{*}{$\begin{array}{l}\text { T1ststg } \\
\text { middle }\end{array}$} & \multirow{2}{*}{$\begin{array}{l}\text { Tiststg } \\
\text { inner }\end{array}$} & \multirow{2}{*}{$\begin{array}{l}\text { T2ndstg } \\
\text { middle }\end{array}$} \\
\hline & Tdccin & Tdccout & Pin dce & DP1stdcc & & & & & \\
\hline Time & $T 1-20$ & TH-22 & $\mathrm{Pl} 33$ & PDI 33 & $\mathrm{POI}-34$ & T1-37 & $\mathrm{Tl} 30$ & $\pi 1-32$ & $T 1-31$ \\
\hline $10: 20$ & 5.67 & 11.53 & 1364 & 16 & 59 & 11.77 & 11.71 & 11.79 & 11.29 \\
\hline $10: 21$ & 5.65 & 11.53 & 1362 & 16 & 58 & 11.76 & 11.66 & 11.8 & 11.3 \\
\hline $10: 22$ & 5.64 & 11.55 & 1362 & 16 & 59 & 11.73 & 11.66 & 11.79 & 11.27 \\
\hline $10: 23$ & 5.66 & 11.53 & 1364 & 16 & 58 & 11.76 & 11.71 & 11.82 & 11.29 \\
\hline $10: 24$ & 5.64 & 11.53 & 1363 & 16 & 61 & 11.75 & 11.72 & 11.8 & 11.29 \\
\hline $10: 25$ & 5.64 & 11.52 & 1363 & 16 & 61 & 11.73 & 11.71 & 11.8 & 11.29 \\
\hline $10: 26$ & 5.65 & 11.51 & 1362 & 16 & 60 & 11.76 & 11.71 & 11.81 & 11.25 \\
\hline 10.27 & 5.65 & 11.49 & 1364 & 16 & 62 & 11.74 & 11.72 & 11.82 & 11.28 \\
\hline $10: 28$ & 5.66 & 11.49 & 1361 & 16 & 60 & 11.72 & 11.68 & 11.8 & 11.25 \\
\hline $10: 29$ & 5.65 & 11.49 & 1362 & 16 & 58 & 11.73 & 11.68 & 11.78 & 11.3 \\
\hline 10.30 & 5.65 & 11.49 & 1361 & 17 & 57 & 11.72 & 11.68 & 11.8 & 11.3 \\
\hline 1031 & 5.66 & 11.49 & 1363 & 16 & 60 & 11.74 & 11.7 & 11.8 & 11.31 \\
\hline 10.32 & 5.66 & 11.48 & 1362 & 16 & 59 & 11.75 & 11.71 & 11.8 & 11.29 \\
\hline 10.33 & 5.66 & 11.48 & 1363 & 17 & 63 & 11.74 & 11.71 & 11.82 & 11.28 \\
\hline $10: 34$ & 5.65 & 11.48 & 1363 & 16 & 63 & 11.76 & 11.73 & 11.83 & 11.28 \\
\hline 10.35 & 5.63 & 11.51 & 1361 & 16 & 63 & 11.74 & 11.72 & 11.8 & 11.28 \\
\hline $10: 36$ & 5.62 & 11.49 & 1361 & 17 & 59 & 11.73 & 11.72 & 11.76 & 11.26 \\
\hline $10: 37$ & 5.6 & 11.47 & 1359 & 17 & 61 & 11.73 & 11.7 & 11.78 & 11.28 \\
\hline $10: 38$ & 5.61 & 11.48 & 1361 & 17 & 58 & 11.73 & 11.7 & 11.76 & 11.3 \\
\hline 10.39 & 5.62 & 11.47 & 1362 & 17 & 61 & 11.72 & 11.7 & 11.78 & 11.28 \\
\hline $10: 40$ & 5.62 & 11.47 & 1361 & 16 & 64 & 11.7 & 11.7 & 11.77 & 11.27 \\
\hline $10: 41$ & 5.62 & 11.47 & 1363 & 16 & 64 & 11.7 & 11.69 & 11.78 & 11.28 \\
\hline 10.42 & 5.63 & 11.48 & 1363 & 16 & 64 & 11.66 & 11.68 & 11.77 & 11.24 \\
\hline $10: 43$ & 5.65 & 11.48 & 1361 & 16 & 60 & 11.68 & 11.67 & 11.79 & 11.27 \\
\hline 10.44 & 5.66 & 11.47 & 1361 & 16 & 58 & 11.68 & 11.66 & 11.78 & 11.26 \\
\hline 10.45 & 5.65 & 11.44 & 1359 & 17 & 59 & 11.65 & 11.59 & 11.76 & 11.23 \\
\hline $10: 46$ & 5.66 & 11.47 & 1360 & 17 & 57 & 11.69 & 11.61 & $11 . \sqrt{5}$ & 11.24 \\
\hline $10: 47$ & 5.67 & 11.47 & 1361 & 16 & 59 & 11.67 & 11.59 & 11.74 & 11.21 \\
\hline 10.48 & 5.69 & 11.46 & 1361 & 16 & 63 & 11.61 & 11.62 & 11.73 & 11.18 \\
\hline $10: 49$ & 5.68 & 11.46 & 1362 & 16 & 61 & 11.56 & 11.61 & 11.69 & 11.17 \\
\hline 1050 & 5.69 & 11.46 & 1359 & 16 & 59 & 11.57 & 1156 & 11.7 & 11.17 \\
\hline 10.51 & 5.66 & 11.48 & 1360 & 17 & 58 & 11.58 & 11.56 & 11.67 & 11.2 \\
\hline 1052 & 5.67 & 11.47 & 1359 & 17 & 57 & 11.53 & 11.56 & 11.65 & 11.18 \\
\hline 10.53 & 5.69 & 11.46 & 1360 & 17 & 57 & 11.56 & 11.52 & 11.66 & 11.17 \\
\hline $10: 54$ & 5.68 & 11.46 & 1361 & 17 & 61 & 11.58 & 11.5 & 11.67 & 11.13 \\
\hline 1055 & 5.68 & 11.45 & 1361 & 17 & 60 & 11.56 & 11.5 & 11.69 & 11.11 \\
\hline 10.56 & 5.69 & 11.46 & 1360 & 17 & 57 & 11.53 & 11.49 & 11.69 & 11.13 \\
\hline $10: 57$ & 5.69 & 11.45 & 1357 & 17 & 57 & 11.52 & 11.5 & 11.64 & 11.15 \\
\hline $10: 58$ & 5.68 & 11.47 & 1359 & 17 & 56 & 11.53 & 11.44 & 11.6 & 11.12 \\
\hline 10.59 & 5.68 & 11.47 & 1359 & 16 & 61 & 11.51 & 11.42 & 11.61 & 11.13 \\
\hline 1100 & 5.68 & 11.45 & 1360 & 16 & 60 & 11.55 & 11.43 & 11.65 & 11.11 \\
\hline 11.01 & 5.68 & 11.43 & 1361 & 16 & 59 & 11.54 & 11.45 & 11.66 & 11.11 \\
\hline $11: 02$ & 5.69 & 11.39 & 1362 & 16 & 59 & 11.54 & 11.43 & 11.63 & 11.11 \\
\hline $11: 03$ & 5.67 & 11.46 & 1362 & 16 & 62 & 11.53 & 11.41 & 11.64 & 11.11 \\
\hline $11: 04$ & 5.67 & 11.48 & 1362 & 16 & 62 & 11.53 & 11.42 & 11.66 & 11.1 \\
\hline 11.05 & 5.66 & 11.47 & 1363 & 16 & 60 & 11.53 & 11.42 & 11.64 & 11.1 \\
\hline 11.06 & 5.68 & 11.47 & 1364 & 16 & 63 & 11.52 & 11.39 & 11.62 & 11.1 \\
\hline 11.07 & 5.68 & 11.49 & 1363 & 16 & 61 & 11.55 & 11.4 & 11.64 & 11.09 \\
\hline $11: 08$ & 5.7 & 11.49 & 1364 & 16 & 61 & 11.53 & 11.37 & 11.65 & 11.06 \\
\hline $11: 00$ & 5.71 & 11.49 & 1363 & 15 & $\infty$ & 11.47 & 11.35 & 11.61 & 11.06 \\
\hline $11: 10$ & 5.69 & 11.48 & 1362 & 16 & $\infty$ & 11.46 & 11.35 & 11.58 & 11.07 \\
\hline $11: 11$ & 560 & 11.5 & 1364 & 16 & 58 & 11.46 & 11.35 & 11.57 & 11.07 \\
\hline $11: 12$ & 569 & 11.49 & 1364 & 16 & 60 & 11.44 & 11.36 & 11.59 & 11.05 \\
\hline $11: 13$ & 5.68 & 11.49 & 1364 & 16 & 58 & 11.47 & 11.37 & 11.6 & 11.03 \\
\hline $11: 14$ & 5.69 & 11.48 & 1364 & 16 & 61 & 11.51 & 11.31 & 11.58 & 10.99 \\
\hline $11: 15$ & 5.7 & 11.48 & 1365 & 16 & 61 & 11.47 & 11.29 & 11.58 & 11.02 \\
\hline $11: 16$ & 5.7 & 11.51 & 1366 & 16 & 59 & 11.47 & 11.28 & 11.58 & 11.03 \\
\hline $11: 17$ & 5.71 & 11.5 & 1368 & 16 & 66 & 11.47 & 11.31 & 11.63 & 11.05 \\
\hline $11: 18$ & 5.7 & 11.52 & 1366 & 16 & 60 & 11.5 & 11.3 & 11.63 & 11.03 \\
\hline $11: 19$ & 5.71 & 11.52 & 1366 & 16 & 60 & 11.5 & 11.29 & 11.55 & 11.04 \\
\hline Average & 5.67 & 11.48 & 1362 & 16 & 60 & 11.61 & 11.55 & 11.71 & 11.18 \\
\hline Stdev & 0.00 & 0.03 & 2 & 10 & 2 & 0.11 & 0.15 & 0.08 & 0.1 \\
\hline
\end{tabular}


Vacuum Pump Life Test for the $210 \mathrm{~kW}$ OC-OTEC Experimental Plant

\section{Appendix A}

POWER PRODUCTION (KW)

\begin{tabular}{|c|c|c|c|c|}
\hline \multicolumn{5}{|c|}{ Time History Record Typical Nov. Conditions } \\
\hline & PWgross & PWesstial & WPnones. & PWnet \\
\hline \multicolumn{5}{|c|}{ 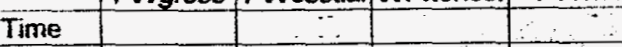 } \\
\hline $10: 20$ & 192.5 & 137.4 & 9.8 & $\$ 5.4$ \\
\hline $10: 21$ & 191.7 & 137.2 & 9.8 & 55 \\
\hline $10: 22$ & 191.9 & 136.9 & 9.8 & 55.4 \\
\hline $10: 23$ & 192.7 & 136.8 & 9.8 & 56.3 \\
\hline $10: 24$ & 191.7 & 136.7 & 9.8 & 56.4 \\
\hline 10.25 & 191.7 & 136.7 & 9.9 & 55.5 \\
\hline $10: 26$ & 190.6 & 136.7 & 9.8 & 54.2 \\
\hline $10: 27$ & 191.7 & 136.9 & 9.8 & 55.4 \\
\hline $10: 28$ & 192.4 & 136.8 & 9.9 & 55.9 \\
\hline 1029 & 1922 & 137.1 & 9.8 & 55.5 \\
\hline $10: 30$ & 191.8 & 136.9 & 9.9 & 55.3 \\
\hline 10.31 & 192.8 & 141.2 & 9.9 & 51.9 \\
\hline $10: 32$ & 192 & 141.4 & 9.9 & 51 \\
\hline $10: 33$ & 193 & 137 & 9.8 & 56.5 \\
\hline $10: 34$ & 191.8 & 136.7 & 9.9 & 55.6 \\
\hline $10: 35$ & 192 & 137 & 9.8 & 55.5 \\
\hline $10: 36$ & 192.1 & 137 & 99 & 55.4 \\
\hline $10: 37$ & 193.6 & 137 & 9.9 & 57 \\
\hline 10.38 & 193.2 & 136.6 & 9.9 & 57 \\
\hline 1039 & 192.5 & 136.7 & 9.9 & 56.2 \\
\hline $10: 40$ & 192.2 & 136.5 & 10 & 56 \\
\hline $10: 41$ & 1926 & 1365 & 9.9 & 56.5 \\
\hline $10: 42$ & 1922 & 137 & 9.9 & 55.7 \\
\hline 10.43 & 191.8 & 136.7 & 10 & 55.4 \\
\hline $10: 44$ & 1921 & 136.9 & 9.9 & 55.7 \\
\hline $10: 45$ & 191.6 & 137 & 9.9 & 56.1 \\
\hline $10: 46$ & 192.6 & 136.8 & 9.9 & 56.4 \\
\hline 10.47 & 191.4 & 136.4 & 10 & 554 \\
\hline $10: 48$ & 191.2 & 136.5 & 9.9 & 55.2 \\
\hline $10: 49$ & 191 & 137 & 9.9 & 54.3 \\
\hline $10: 50$ & 191 & 136.7 & 9.9 & 548 \\
\hline 10.51 & 191.3 & 141.3 & 9.9 & 50.5 \\
\hline $10: 52$ & 191.2 & 141.5 & 9.9 & 50.2 \\
\hline $10: 53$ & 191 & 137.6 & 9.9 & 54 \\
\hline $10: 54$ & 191.2 & 136.3 & 9.9 & 55.4 \\
\hline $10: 55$ & 190.8 & 137.1 & 9.9 & 54 \\
\hline 10.56 & 191.3 & 137.1 & 9.9 & 54.7 \\
\hline 10.57 & 191.4 & 136.9 & 9.9 & 55 \\
\hline 10.58 & 191.6 & 136.5 & 9.9 & 55.6 \\
\hline $10: 59$ & 191.9 & 136.3 & 9.9 & 56.1 \\
\hline $11: 00$ & 191.8 & 136.2 & 9.9 & 56.1 \\
\hline $11: 01$ & 191.4 & 136.4 & 9.9 & 55.5 \\
\hline 11.02 & 190.7 & 136.4 & 9.8 & 54.8 \\
\hline 11.03 & 191.3 & 136.3 & 9.9 & 55.5 \\
\hline 11.04 & 189.9 & 136.5 & 9.9 & 54 \\
\hline 11.05 & 191.2 & 136.5 & 9.9 & 56.1 \\
\hline $11: 06$ & 190.6 & 136.4 & 9.9 & 54.8 \\
\hline 11.07 & 190.8 & 136.2 & 9.8 & 55.1 \\
\hline 11.08 & 190.9 & 136.3 & 9.9 & 55 \\
\hline 11.00 & 190.6 & 136.5 & 9.9 & 54.6 \\
\hline $11: 10$ & 191.1 & 136.3 & 9.9 & 55.2 \\
\hline $11: 11$ & 191 & 141 & 9.9 & 50.6 \\
\hline $11: 12$ & 191.8 & 141.2 & 10.6 & 51.2 \\
\hline $11: 13$ & 191.3 & 137.3 & 10.2 & 54.4 \\
\hline $11: 14$ & 191.2 & 136.3 & 10.4 & 55.1 \\
\hline $11: 15$ & 191.4 & 137.4 & 9.9 & 54.6 \\
\hline $11: 16$ & 190.3 & 136.1 & 9.9 & 54.5 \\
\hline $11: 17$ & 190.4 & 136.1 & 9.9 & 54.6 \\
\hline $11: 18$ & 189.9 & 136.7 & 9.9 & 53.6 \\
\hline $11: 19$ & 191.2 & 136.8 & 9.9 & 55 \\
\hline Average & 191.6 & 137.2 & 9.9 & 54.8 \\
\hline Stdev & 0.8 & 1.4 & 0.1 & 1.5 \\
\hline
\end{tabular}


Vacuum Pump Life Test for the $210 \mathrm{~kW}$ OC-OTEC Experimental Plant

Appendix A

VACUUM COMPRESSOR SUBSYSTEM PARAMETERS

\begin{tabular}{|c|c|c|c|c|c|c|c|c|c|c|c|c|}
\hline \multicolumn{13}{|c|}{ Time History Record ( 60 minutes long) Typical Nov. Conditions } \\
\hline & Pinfst & Pin2nd & Pin3rd & Pin 4th & Pinsth & Mnc,g/s & AMP & RPM & AMP & RPM & AMP & RPM \\
\hline Time & PI-105 & PI-104 & Pl-103 & $\mathrm{Pl}-102$ & PI-101 & $F 1-60$ & AMP-1A & RPM-1A & AMP-1B & RPM-1B & AMP-1C & RPM-1C \\
\hline $10: 20$ & 1299 & 2154 & 4487 & 8717 & 8374 & 18.5 & 4.5 & 25798 & 4.4 & 25798 & 4.4 & 25798 \\
\hline $10: 21$ & 1299 & 2163 & 4506 & 8762 & 8420 & 18.7 & 4.4 & 25797 & 4.4 & 25797 & 4.4 & 25797 \\
\hline $10: 22$ & 1299 & 2164 & 4509 & 8766 & 8418 & 18.6 & 4.4 & 25797 & 4.4 & 25797 & 4.4 & 25797 \\
\hline $10: 23$ & 1301 & 2161 & 4501 & 8745 & 8401 & 18.6 & 4.4 & 25799 & 4.4 & 25799 & 4.4 & 25799 \\
\hline $10: 24$ & 1300 & 2166 & 4511 & 8754 & 8413 & 18.6 & 4.4 & 25797 & 4.4 & 25797 & 4.3 & 25797 \\
\hline $10: 25$ & 1298 & 2154 & 4487 & 8720 & 8376 & 18.5 & 4.4 & 25796 & 4.4 & 25796 & 4.3 & 25796 \\
\hline $10: 26$ & 1300 & 2167 & 4516 & 8778 & 8432 & 18.6 & 4.4 & 25795 & 4.4 & 25795 & 4.3 & 25796 \\
\hline $10: 27$ & 1300 & 2164 & 4513 & 8770 & 8426 & 18.7 & 4.4 & 25795 & 4.4 & 25795 & 4.3 & 25795 \\
\hline $10: 28$ & 1298 & 2148 & 4481 & 8708 & 8369 & 18.5 & 4.4 & 25801 & 4.4 & 25801 & 4.4 & 25801 \\
\hline $10: 29$ & 1301 & 2156 & 4505 & 8746 & 8406 & 18.6 & 4.4 & 25799 & 4.4 & 25799 & 4.4 & 25799 \\
\hline $10: 30$ & 1300 & 2158 & 4509 & 8760 & 8418 & 18.6 & 4.4 & 25803 & 4.4 & 25803 & 4.3 & 25803 \\
\hline $10: 31$ & 1300 & 2150 & 4500 & 8714 & 8369 & 18.4 & 4.4 & 25798 & 4.4 & 25798 & 4.4 & 25798 \\
\hline $10: 32$ & 1302 & 2169 & 4539 & 8788 & 8445 & 18.6 & 4.4 & 25800 & 4.4 & 25800 & 4.3 & 25800 \\
\hline $10: 33$ & 1297 & 2147 & 4498 & 8703 & 8362 & 18.4 & 4.4 & 25799 & 4.4 & 25799 & 4.3 & 25790 \\
\hline $10: 34$ & 1300 & 2166 & 4531 & 8800 & 8453 & 18.6 & 4.4 & 25800 & 4.4 & 25800 & 4.3 & 25800 \\
\hline $10: 35$ & 1298 & 2149 & 4501 & 8714 & 8371 & 18.4 & 4.4 & 25797 & 4.4 & 25797 & 4.3 & 25797 \\
\hline $10: 36$ & 1301 & 2168 & 4532 & 8805 & 8458 & 18.6 & 4.4 & 25796 & 4.4 & 25796 & 4.3 & 25796 \\
\hline $10: 37$ & 1297 & 2146 & 4485 & 8707 & 8366 & 18.4 & 4.4 & 25799 & 4.4 & 25799 & 4.3 & 25799 \\
\hline $10: 38$ & 1299 & 2159 & 4513 & 8767 & 8423 & 18.4 & 4.4 & 25793 & 4.4 & 25793 & 4.4 & 25793 \\
\hline 10.39 & 1296 & 2158 & 4512 & 8760 & 8420 & 18.4 & 4.4 & 25801 & 4.4 & 25801 & 4.3 & 25801 \\
\hline $10: 40$ & 1293 & 2152 & 4494 & 8731 & 8391 & 18.4 & 4.4 & 25800 & 4.4 & 25800 & 4.3 & 25800 \\
\hline $10: 41$ & 1292 & 2152 & 4494 & 8727 & 8384 & 18.3 & 4.4 & 25800 & 4.4 & 25800 & 4.4 & 25800 \\
\hline $10: 42$ & 1293 & 2154 & 4497 & 8735 & 8392 & 18.3 & 4.4 & 25798 & 4.4 & 25798 & 4.4 & 25798 \\
\hline $10: 43$ & 1291 & 2146 & 4474 & 8687 & 8352 & 18.2 & 4.4 & 25799 & 4.4 & 25799 & 4.4 & 25799 \\
\hline $10: 44$ & 1293 & 2163 & 4509 & 8755 & 8412 & 18.3 & 4.4 & 25800 & 4.4 & 25800 & 4.4 & 25800 \\
\hline 10.45 & 1291 & 2148 & 4469 & 8679 & 8342 & 18.2 & 4.4 & 25804 & 4.4 & 25804 & 4.4 & 25804 \\
\hline $10: 46$ & 1293 & 2159 & 4494 & 8730 & 8390 & 18.3 & 4.4 & 25800 & 4.4 & 25800 & 4.4 & 25800 \\
\hline $10: 47$ & 1293 & 2163 & 4500 & 8743 & 8401 & 18.4 & 4.4 & 25800 & 4.4 & 25800 & 4.4 & 25800 \\
\hline $10: 48$ & 1289 & 2154 & 4471 & 8690 & 8355 & 18.3 & 4.4 & 25802 & 4.4 & 25802 & 4.4 & 25802 \\
\hline $10: 49$ & 1292 & 2168 & 4507 & 8756 & 8413 & 18.2 & 4.4 & 25802 & 4.4 & 25802 & 4.4 & 25802 \\
\hline $10: 50$ & 1290 & 2161 & 4488 & 8706 & 8368 & 18.3 & 4.4 & 25802 & 4.4 & 25802 & 4.3 & 25802 \\
\hline 10.51 & 1293 & 2155 & 4478 & 8688 & 8349 & 18.2 & 4.4 & 25798 & 4.4 & 25798 & 4.4 & 25798 \\
\hline $10: 52$ & 1293 & 2166 & 4504 & 8725 & 8384 & 18.3 & 4.4 & 25802 & 4.4 & 25802 & 4.4 & 25802 \\
\hline 10.53 & 1293 & 2180 & 4526 & 8762 & 8421 & 18.4 & 4.4 & 25802 & 4.4 & 25802 & 4.3 & 25802 \\
\hline $10: 54$ & 1288 & 2156 & 4483 & 8682 & 8343 & 18.2 & 4.4 & 25803 & 4.4 & 25803 & 4.4 & 25803 \\
\hline $10: 56$ & 1291 & 2167 & 4507 & 8754 & 8411 & 18.3 & 4.4 & 25802 & 4.4 & 25802 & 4.3 & 25802 \\
\hline $10: 56$ & 1292 & 2167 & 4508 & 8738 & 8397 & 18.2 & 4.4 & 25803 & 4.4 & 25803 & 4.4 & 25803 \\
\hline $10: 57$ & 1287 & 2157 & 4501 & 8736 & 8398 & 18.3 & 4.4 & 25802 & 4.4 & 25802 & 4.4 & 25802 \\
\hline $10: 58$ & 1291 & 2168 & 4517 & 8756 & 8410 & 18.2 & 4.4 & 25801 & 4.4 & 25801 & 4.4 & 25801 \\
\hline $10: 59$ & 1284 & 2141 & 4498 & 8729 & 8389 & 18.3 & 4.4 & 25801 & 4.4 & 25801 & 4.4 & 25801 \\
\hline $11: 00$ & 1286 & 2158 & 4490 & 8722 & 8381 & 18.2 & 4.4 & 25807 & 4.4 & 25807 & 4.4 & 25807 \\
\hline $11: 01$ & 1288 & 2167 & 4508 & 8764 & 8421 & 18.3 & 4.4 & 25800 & 4.4 & 25800 & 4.3 & 25800 \\
\hline $11: 02$ & 1289 & 2169 & 4514 & 8753 & 8412 & 18.3 & 4.4 & 25801 & 4.4 & 25801 & 4.4 & $25801^{\circ}$ \\
\hline $11: 03$ & 1286 & 2152 & 4488 & 8684 & 8345 & 18.1 & 4.4 & 25801 & 4.4 & 25801 & 4.4 & 25801 \\
\hline $11: 04$ & 1289 & 2164 & 4513 & 8753 & 8410 & 18.3 & 4.4 & 25801 & 4.4 & 25801 & 4.3 & 25801 \\
\hline 11.05 & 1288 & 2152 & 4486 & 8716 & 8375 & 18.1 & 4.4 & 25797 & 4.4 & 25797 & 4.4 & 25797 \\
\hline 11.06 & 1288 & 2161 & 4502 & 8753 & 8412 & 18.2 & 4.4 & 25801 & 4.4 & 25801 & 4.4 & 25801 \\
\hline 11.07 & 1285 & 2140 & 4463 & 8669 & 8333 & 18 & 4.4 & 25797 & 4.4 & 25797 & 4.4 & 25797 \\
\hline 11.08 & 1287 & 2164 & 4516 & 8751 & 8409 & 18.2 & 4.4 & 25802 & 4.4 & 25802 & 4.4 & 25802 \\
\hline $11: 09$ & 1287 & 2161 & 4516 & 8739 & 8397 & 18.1 & 4.4 & 25799 & 4.4 & 25799 & 4.4 & 25799 \\
\hline $11: 10$ & 1285 & 2156 & 4495 & 8701 & 8368 & 18.2 & 4.4 & 25802 & 4.4 & 25802 & 4.4 & 25802 \\
\hline $11: 11$ & 1289 & 2168 & 4518 & 8747 & 8410 & 18.2 & 4.4 & 25799 & 4.4 & 25799 & 4.4 & 25799 \\
\hline $11: 12$ & 1287 & 2153 & 4482 & 8682 & 8348 & 18.1 & 4.5 & 25804 & 4.4 & 25804 & 4.4 & 25804 \\
\hline $11: 13$ & 1290 & 2174 & 4528 & 8791 & 8452 & 18.3 & 4.4 & 25802 & 4.4 & 25802 & 4.4 & 25802 \\
\hline $11: 14$ & 1285 & 2146 & 4463 & 8674 & 8343 & 18.1 & 4.5 & 25802 & 4.4 & 25802 & 4.4 & 25802 \\
\hline $11: 15$ & 1287 & 2158 & 4491 & 8728 & 8392 & 18.2 & 4.5 & 25804 & 4.4 & 25804 & 4.4 & 25804 \\
\hline 11:16 & 1290 & 2176 & 4526 & 8799 & 8462 & 18.3 & 4.4 & 25800 & 4.4 & 25800 & 4.4 & 25800 \\
\hline $11: 17$ & 1284 & 2149 & 4469 & 8683 & 8348 & 18.2 & 4.4 & 25798 & 4.4 & 25798 & 4.4 & 25798 \\
\hline $11: 18$ & 1291 & 2175 & 4527 & 8800 & 8460 & 18.4 & 4.4 & 25800 & 4.4 & 25800 & 4.4 & 25800 \\
\hline $11: 19$ & 1289 & 2143 & 4460 & 8666 & 8330 & 18.1 & 4.5 & 25799 & 4.4 & 25799 & 4.4 & 25799 \\
\hline Average & 1293 & 2150 & 4500 & 8734 & 8394 & 18.3 & 4.4 & 25800 & 4.4 & 25800 & 4.4 & 25800 \\
\hline $\mathrm{ev}$ & 5 & 9 & 18 & 6 & 4 & 2 & 0.0 & & 0 & & 0 & 13 \\
\hline
\end{tabular}


Vacuum Pump Life Test for the $210 \mathrm{~kW}$ OC-OTEC Experimental Plant

Appendix A

VACUUM COMPRESSOR SUBSYSTEM PARAMETERS

\begin{tabular}{|c|c|c|c|c|c|c|}
\hline \multicolumn{7}{|c|}{ Time History Record (60 minutes long) Typical Nov. Conditions } \\
\hline & AMP & RPM & AMP & RPM & RPM & AMP \\
\hline Time & AMP-2 & RPM-2 & AMP-3 & RPM-3 & RPM-5B & AMP $5 B$ \\
\hline $10: 20$ & 7.3 & 27784 & 6.3 & 35995 & 4149 & 28.6 \\
\hline $10: 21$ & 7.3 & 27781 & 6.3 & 35987 & 4148 & 28.6 \\
\hline $10: 22$ & 7.3 & 27786 & 6.3 & 35989 & 4147 & 28.6 \\
\hline $10: 23$ & 7.3 & 27781 & 6.3 & 36990 & 4148 & 28.7 \\
\hline $10: 24$ & 7.3 & 27784 & 6.3 & 35906 & 4148 & 28.7 \\
\hline $10: 25$ & 7.3 & 27789 & 6.3 & 35990 & 4147 & 28.6 \\
\hline $10: 26$ & 7.3 & 27783 & 6.3 & 36000 & 4147 & 28.7 \\
\hline $10: 27$ & 7.3 & 27791 & 6.3 & 36000 & 4147 & 28.7 \\
\hline $10: 28$ & 7.3 & 27791 & 6.2 & 35998 & 4147 & 28.6 \\
\hline $10: 29$ & 7.3 & 27785 & 6.3 & 35992 & 4148 & 28.7 \\
\hline $10: 30$ & 7.3 & 27785 & 6.3 & 35995 & 4147 & 28.7 \\
\hline $10: 31$ & 7.3 & 27785 & 6.3 & 35992 & 4147 & 28.6 \\
\hline $10: 32$ & 7.3 & 27786 & 6.3 & 35996 & 4147 & 28.7 \\
\hline $10: 33$ & 7.3 & 27785 & 6.2 & 36987 & 4148 & 28.6 \\
\hline $10: 34$ & 7.4 & 27790 & 6.3 & 36003 & 4148 & 28.6 \\
\hline $10: 35$ & 7.3 & 27788 & 6.3 & 35988 & 4147 & 28.6 \\
\hline $10: 36$ & 7.4 & 27791 & 6.3 & 35991 & 4147 & 28.7 \\
\hline $10: 37$ & 7.3 & 27787 & 6.3 & 35989 & 4147 & 28.7 \\
\hline 10.38 & 7.3 & 27785 & 6.3 & 36000 & 4147 & 28.7 \\
\hline $10: 39$ & 7.3 & 27781 & 6.3 & 35995 & 4147 & 28.7 \\
\hline $10: 40$ & 7.3 & 27791 & 6.3 & 35997 & 4147 & 28.6 \\
\hline $10: 41$ & 7.3 & 27789 & 6.3 & 35997 & 4147 & 28.7 \\
\hline $10: 42$ & $7 . \overline{3}$ & 27783 & 6.3 & 35990 & 4147 & 28.6 \\
\hline $10: 43$ & 7.3 & 27789 & 6.3 & 35988 & 4147 & 28.6 \\
\hline $10: 44$ & 7.3 & 27788 & 6.3 & 35994 & 4147 & 28.7 \\
\hline $10: 45$ & 7.3 & 27783 & 6.2 & 35987 & 4147 & 28.6 \\
\hline $10: 46$ & 7.3 & 27785 & 6.3 & 36001 & 4147 & 28.6 \\
\hline $10: 47$ & 7.3 & 27785 & 6.3 & 35989 & $41 \overline{47}$ & 28.6 \\
\hline $10: 48$ & 73 & 27791 & 62 & 35998 & 4147 & 28.6 \\
\hline $10: 49$ & 7.3 & 27790 & 6.3 & 35997 & 4147 & 28.6 \\
\hline $10: 50$ & 7.3 & 27791 & 6.3 & 36004 & 4147 & 28.5 \\
\hline $10: 51$ & 7.3 & 27786 & 6.3 & 35996 & 4147 & 28.6 \\
\hline 10.52 & 7.3 & 27792 & 6.3 & 35992 & 4146 & 28.6 \\
\hline $10: 53$ & 7.3 & 27792 & 6.3 & 35996 & 4146 & 28.6 \\
\hline $10: 54$ & 7.3 & 27788 & 6.3 & 36003 & 4145 & 28.6 \\
\hline $10: 55$ & 7.3 & 27790 & 6.3 & 36001 & 4145 & 28.6 \\
\hline 10.56 & 7.3 & 27794 & 6.3 & 36005 & 4146 & 28.6 \\
\hline 10.57 & 7.2 & 27792 & 6.3 & 35994 & 4145 & 28.6 \\
\hline 10.58 & 7.3 & 27789 & 6.3 & 36998 & 4145 & 28.6 \\
\hline 10.50 & 7.2 & 27787 & 6.3 & 36998 & 4145 & 28.6 \\
\hline $11: 00$ & 7.3 & 27786 & 6.3 & 35990 & 4145 & 28.5 \\
\hline $11: 01$ & 7.3 & 27787 & 6.3 & 35991 & 4145 & 28.6 \\
\hline $11: 02$ & 7.3 & 27788 & 6.3 & 35997 & 4145 & 28.6 \\
\hline 11.03 & 7.3 & 27788 & 6.3 & 35993 & 4145 & 28.6 \\
\hline $11: 04$ & 7.3 & 27788 & 6.3 & 35986 & 4145 & 28.6 \\
\hline $11: 05$ & 7.3 & 27794 & 6.3 & 36008 & 4145 & 28.6 \\
\hline $11: 06$ & 7.3 & 27784 & 6.3 & 36992 & 4146 & 28.6 \\
\hline 11.07 & 7.3 & 27788 & 6.3 & 36000 & 4146 & 28.6 \\
\hline 11.08 & 7.3 & 27788 & 6.3 & 35997 & 4147 & 28.6 \\
\hline $11: \infty 9$ & 7.3 & 27786 & 6.3 & 35995 & 4145 & 28.6 \\
\hline $11: 10$ & 7.3 & 27787 & 6.3 & 35978 & 4145 & 28.6 \\
\hline $11: 11$ & 7.4 & 27789 & 6.3 & 36998 & 4145 & 28.6 \\
\hline $11: 12$ & 7.3 & 27787 & 63 & 35987 & 4145 & 28.6 \\
\hline $11: 13$ & 7.4 & 27784 & 6.3 & 35988 & 4145 & 28.6 \\
\hline $11: 14$ & 7.3 & 27785 & 6.3 & 35089 & 4145 & 28.6 \\
\hline $11: 15$ & 7.3 & 27789 & 6.3 & 35997 & 4145 & 28.6 \\
\hline $11: 16$ & 7.3 & 27788 & 6.3 & 35982 & 4145 & 28.6 \\
\hline $11: 17$ & 7.3 & 27787 & 6.3 & 35986 & 4146 & 28.6 \\
\hline $11: 18$ & 7.4 & 27784 & 6.4 & 35096 & 4146 & 28.6 \\
\hline $11: 19$ & 7.3 & 27786 & 6.3 & 35990 & 4146 & 28.6 \\
\hline Average & 7.3 & 27787 & 16.3 & 35994 & 4147 & 28.6 \\
\hline Stdev & 10 & 3 & 10.0 & 6 & 1 & 0 \\
\hline
\end{tabular}




\section{Appendix B}

\section{UNCERTAINTY OF DERIVED PARAMETERS}

\section{Power Production Mode}

This Appendix discusses the uncertainty of the parameters routinely derived at the 210 OC-OTEC Experimental Facility. The following end-toend precisions were specified for the primary measurements at the design point:

Temperatures

Steam Pressures

Water Flowrates

Mass Flowrate NC

Power $\pm 0.05^{\circ} \mathrm{C}$

$\pm 5 \mathrm{~Pa}$

$\pm 100 \mathrm{gpm}$

$\pm 1 \mathrm{~g} / \mathrm{s}$

$\pm 1 \mathrm{~kW}$ 
OC-OTEC Vacuum Pumps Life Cycle Tests

Appendix B

\section{Table of Contents}

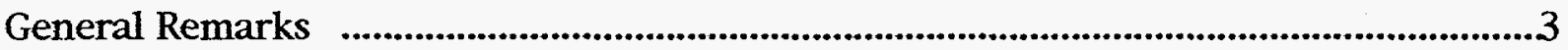

Evaporator Effectiveness

Direct Contact Condenser (DCC) Effectiveness ...........................................................6

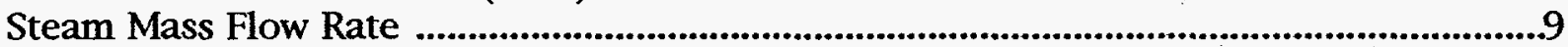

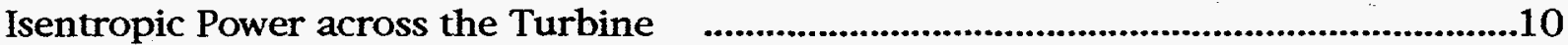

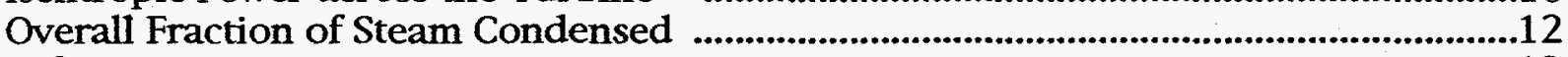

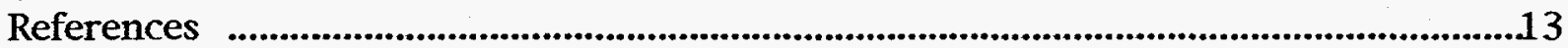


Appendix B

\section{UNCERTAINTY OF DERIVED PARAMETERS}

\section{General Remarks}

The conceptual discussion below summarizes the ideas presented in Benedict (1984).

The measurement of any physical parameter is tainted by sources of error traditionally assigned to two types: a systematic bias, and a random error. Use of these concepts implies that the measurement is actually performed $\mathrm{N}$ times for a given input.

The average value $\mu$, taken over the set of $N$ readings $X_{k}$, is the estimator of the physical parameter, and the separation between $\mu$ and the true value is called bias, $B$. Strictly speaking, good accuracy is associated with small bias. Moreover, a 'perfect' instrument calibration, prior to the performance of the desired measurements, would actually eliminate any bias.

Similarly, the scatter of the $\mathrm{N}$ readings, away from their average, results from random errors, and strictly speaking, good precision is associated with small such errors. To quantify precision, or define confidence intervals, one may use the unbiased estimate of the standard deviation of the set, defined by

$$
S=\sqrt{\frac{1}{N-1} \sum_{k=1}^{N}\left(X_{k}-\mu\right)^{2}} .
$$

$\mathrm{S}$ is multiplied by $\mathrm{t}-1, \mathrm{p}$, the Student $\mathrm{t}$ distribution for $\mathrm{N}-1$ degrees of freedom and probability $p$. If $\mathrm{N}$ becomes very large, the $t$ statistics asymptotically tends to the better-known normal Gaussian distribution. In summary, $\mu$ will belong to the interval $X_{k} \pm t_{N}-1, p S$, defined by any measured value $X_{k}$, with the probability $p$.

It must be noted that unlike random errors, bias errors do not lend themselves to any statistical treatment This presents a dilemma when one wishes to simply quantify the uncertainty $U$ of a measurement, whereby a single confidence interval would encompass both the effect of systematic and random errors. Experience proved that in general, defining $U$ as the sum $B+t \mathrm{~N}-1, p S$ is too conservative. A favored formula is rather:

$$
U=\sqrt{B^{2}+\left(t_{N-1,095} S\right)^{2}}
$$

The simple concepts outlined above, however, are less easily applicable as they seem. In general, the manufacturers of measuring devices do not go into sufficient details for a clear distinction between accuracy and precision. Upon Benedict's recommendation, it may be preferable to consider the uncertainty information provided without statistical qualification, or simply estimated by the performer of the measurements, as systematic error, or bias. On the other hand, any attempt to determine $S$ experimentally is only possible if one can reasonably satisfy the given (fixed) input requirement. 
Therefore, estimated uncertainties $\delta \mathrm{P}_{\mathbf{i}}$, for individual devices measuring the physical parameters $P_{i}$, will be treated as bias. Once again with little statistical justification, the uncertainty $\delta Q$ on a derived parameter $Q\left(P_{i}\right)$, function of $M$ parameters $P_{i}$, is estimated as:

$$
\delta Q=\sqrt{\sum_{i=1}^{M}\left(\frac{\partial Q}{\partial P_{i}} \delta P_{i}\right)^{2}}
$$

Although the expression above cannot be quantified from a probabilistic standpoint like random errors, its use seems to have proved satisfactory on the basis of experience. We will therefore state that the true value of the derived physical parameter $Q$ is 'likely' to belong to the interval $Q \pm \delta Q$. This interval could be restricted further when there is a theoretical reason to do so: e.g., a heat exchanger effectiveness cannot exceed 1.0 (100\%).

Examples will now be given to illustrate the above formula in the context of the $210 \mathrm{~kW}$ OC-OTEC Experimental Apparatus.

\section{Evaporator Effectiveness}

The definition of evaporator effectiveness is provided below:

$$
\varepsilon=\frac{T_{\text {in }}-T_{\text {out }}}{T_{\text {in }}-\left\{T_{\text {sat }}\left(\text { pev }_{\text {ev }}\right)+0.31\right\}}
$$

The baseline values used henceforth are given as $\mathrm{Tin}=26^{\circ} \mathrm{C}$, $\mathrm{T}_{\text {out }}=22.5^{\circ} \mathrm{C}$, $\mathrm{pev}_{\mathrm{ev}}=2620 \mathrm{~Pa}$ and the following curve fit for $\mathrm{T}_{\text {sat, }}$ in ${ }^{\circ} \mathrm{C}$ :

$$
\mathrm{T}_{\text {sat }}(\mathrm{pev})=\frac{4026.9759}{-\log \left\{\frac{\mathrm{pev}-3.74}{161.7574}\right\}+18.4779}-234.7384
$$

This input yields $\mathrm{T}_{\text {sat }}(\mathrm{pev})=21.85^{\circ} \mathrm{C}$ and $\varepsilon=0.91$.

Two alternate ways are considered to experimentally determine $\varepsilon$ : both utilize a pressure reading of $\mathrm{pev}$ and a differential temperature measurement of the numerator, $\Delta \mathrm{T}_{\mathrm{ev}}=\mathrm{T}_{\mathrm{in}}-\mathrm{T}_{\mathrm{out}}$; the inlet water temperature in the numerator, however, can be obtained either a) directly or b) by adding $\Delta \mathrm{T}_{\mathrm{ev}}$ to $\mathrm{T}_{\text {out }}$.

a)

$$
\varepsilon=\frac{\Delta \mathrm{T}_{\text {ev }}}{\mathrm{T}_{\text {in }}-\left\{\mathrm{T}_{\text {sat }}\left(\mathrm{p}_{\text {ev }}\right)+0.31\right\}}
$$

The following sensitivity coefficients must be considered:

$$
\frac{\partial \varepsilon}{\partial T_{\text {in }}}=-\frac{\Delta T_{\text {ev }}}{\left(T_{\text {in }}-\left\{T_{\text {sat }}\left(p_{\text {ev }}\right)+0.31\right\}\right)^{2}}=-\frac{\varepsilon^{2}}{\Delta T_{\text {ev }}} \approx-0.237 \mathrm{~K}^{-1}
$$


Appendix B

$\frac{\partial \varepsilon}{\partial \Delta T_{\text {ev }}}=\frac{1}{T_{\text {in }}-\left\{T_{\text {sat }}\left(p_{\text {ev }}\right)+0.31\right\}}=\frac{\varepsilon}{\Delta T_{\text {ev }}} \approx 0.260 \mathrm{~K}^{-1}$

$\frac{\partial \varepsilon}{\partial \mathrm{pev}_{\mathrm{ev}}}=\frac{\partial \varepsilon}{\partial \mathrm{T}_{\text {sat }}} \frac{\partial \mathrm{T}_{\text {sat }}}{\partial \mathrm{pev}} \approx 0.001481 \mathrm{~Pa}^{-1}$

since $\frac{\partial \varepsilon}{\partial \mathrm{T}_{\text {sat }}}=-\frac{\partial \varepsilon}{\partial \mathrm{T}_{\text {in }}} \approx 0.237 \mathrm{~K}^{-1}$,

and $\frac{\partial \mathrm{T}_{\text {sat }}}{\partial \mathrm{p}_{\mathrm{ev}}}=\frac{4026.9759\left\{\frac{1}{\mathrm{p}_{\mathrm{ev}}-3.74}\right\}}{\left\{-\mathrm{Log} \frac{\mathrm{p}_{\mathrm{ev}}-3.74}{1617574}+18.4779\right\}^{2}}=0.00625 \mathrm{~K} / \mathrm{Pa}$

Moreover, $\delta \varepsilon=\sqrt{\left(\frac{\partial \varepsilon}{\partial \mathrm{T}_{\mathrm{in}}} \delta \mathrm{T}_{\mathrm{in}}\right)^{2}+\left(\frac{\partial \varepsilon}{\partial \Delta \mathrm{T}_{\mathrm{ev}}} \delta \Delta \mathrm{T}_{\mathrm{ev}}\right)^{2}+\left(\frac{\partial \varepsilon}{\partial \mathrm{p}_{\mathrm{ev}}} \delta \mathrm{p}_{\mathrm{ev}}\right)^{2}}$

With $\delta \mathrm{T}_{\text {in }}=0.05^{\circ} \mathrm{C}(\mathrm{TE}-10), \delta \Delta \mathrm{T}_{\mathrm{ev}}=0.02^{\circ} \mathrm{C}(\mathrm{TdT}-11)$ and $\delta \mathrm{p}_{\mathrm{ev}}=5.4 \mathrm{~Pa}(\mathrm{PT}-30)$, one obtains $\delta \varepsilon \approx 0.015$

i.e. $\quad \frac{\delta \varepsilon}{\varepsilon}=1.7 \%$

b) $\quad \varepsilon=\frac{\Delta T_{\text {ev }}}{T_{\text {out }}+\Delta T_{\text {ev }}-\left\{T_{\text {sat }}\left(p_{\text {ev }}\right)+0.31\right\}}$

The following sensitivity coefficients must be considered:

$\frac{\partial \varepsilon}{\partial \mathrm{T}_{\text {out }}}=-\frac{\varepsilon^{2}}{\Delta \mathrm{T}_{\mathrm{ev}}} \approx-0.237 \mathrm{~K}^{-1}$

$\frac{\partial \varepsilon}{\partial \Delta \mathrm{T}_{\mathrm{ev}}}=\frac{\mathrm{T}_{\text {out }}-\left\{\mathrm{T}_{\text {sat }}\left(\mathrm{pev}_{\mathrm{ev}}\right)+0.31\right\}}{\left(\mathrm{T}_{\text {in }}-\left\{\mathrm{T}_{\text {sat }}\left(\mathrm{p}_{\mathrm{ev}}\right)+0.31\right\}\right)^{2}}=\frac{\varepsilon-\varepsilon^{2}}{\Delta \mathrm{T}_{\mathrm{ev}}} \approx 0.023 \mathrm{~K}^{-1}$

$\frac{\partial \varepsilon}{\partial \mathrm{pev}}$ is the same as above.

Moreover, $\delta \varepsilon=\sqrt{\left(\frac{\partial \varepsilon}{\partial \mathrm{T}_{\text {out }}} \delta \mathrm{T}_{\text {out }}\right)^{2}+\left(\frac{\partial \varepsilon}{\partial \Delta \mathrm{T}_{\mathrm{ev}}} \delta \Delta \mathrm{T}_{\mathrm{ev}}\right)^{2}+\left(\frac{\partial \varepsilon}{\partial \mathrm{p}_{\mathrm{ev}}} \delta \mathrm{p}_{\mathrm{ev}}\right)^{2}}$

With $\delta \mathrm{T}_{\text {out }}=0.05^{\circ} \mathrm{C}(\mathrm{TE}-12), \delta \Delta \mathrm{T}_{\mathrm{ev}}=0.02^{\circ} \mathrm{C}(\mathrm{TdT}-11)$ and $\delta \mathrm{pev}_{\mathrm{ev}}=5.4 \mathrm{~Pa}(\mathrm{PT}-30)$, one obtains $\delta \varepsilon \approx 0.014$

i.e. $\quad \frac{\delta \varepsilon}{\varepsilon}=1.6 \%$ 
It is interesting to note that the latter method for determining evaporator effectiveness is slightly more accurate: the presence of $\Delta \mathrm{T}_{\mathrm{ev}}$ in both numerator and denominator, in the definition of $\varepsilon$, reduces the corresponding sensitivity coefficient by one order of magnitude.

\section{Direct Contact Condenser (DCC) Effectiveness}

The definition of the DCC effectiveness mirrors that of the evaporator discussed in the previous section:

$$
\varepsilon D C C=\frac{T_{\text {out }}-\mathrm{T}_{\text {in }}}{\left\{\mathrm{T}_{\text {sat }}\left(\mathrm{p}_{\text {steam }}\right)+0.28\right\}-\mathrm{T}_{\text {in }}},
$$

where psteam is the partial pressure of steam at the inlet of the condenser. Such a definition may be applied separately to the first (co-current) and second (countercurrent) DCC stages, as well as to the overall heat exchanger.

All seawater temperatures are measured directly for the two individual DCC stages (TE-30 and TE-31, respectively), but the temperature rise across the overall heat exchanger is provided by a differential temperature measurement, $\triangle$ TDCC (TdT-21). Consequently, the overall effectiveness should be evaluated from:

$$
\varepsilon \mathrm{DCC}=\frac{\left.\Delta \mathrm{TDCC}_{\text {sat }}\left(\mathrm{p}_{\text {inlet }}\right)+0.28\right\}+\Delta \mathrm{T}_{\mathrm{DCC}}-\mathrm{T}_{\text {out }}}{\left\{\mathrm{T}_{\text {s }}\right.}
$$

For the first-stage and overall DCC, the amount of non-condensibles at the inlet is negligible, and psteam is taken as the overall pressure pinlet, measured in those cases by PT-33. In the absence of a direct temperature measurement for the vapor phase between the two DCC stages, the second-stage inlet partial pressure requires a careful evaluation: calling the total interstage pressure $(p-\Delta p)$, known by the difference between PT-33 and PdT-33, the ratio psteam $/(p-\Delta p)$ is equal to the ratio of steam molar flow rate over total molar flow rate; thus:

$$
\text { psteam }=\frac{(p-\Delta p)}{1+\frac{18}{29} \frac{m_{\text {nc1 }}}{m_{\text {steam }}}}
$$

$\mathrm{m}_{\mathrm{nc}} 1$ represents the mass flow of non-condensibles between DCC stages, and can be estimated by subtracting the estimated release in the second DCC stage alone from the total amount $m_{n c}$, measured by FE-60:

$$
m_{n c 1}=m_{n c}-f_{c w} \rho_{c w} Q_{c w} R_{2 t}
$$

in the above formula, $\mathrm{f}_{\mathrm{CW}}$ is a constant, equal to $18.22 \times 10^{-6} \mathrm{~kg} / \mathrm{kg}$, expressing cold seawater non-condensable content, $\rho_{\mathrm{cw}}$ is the cold seawater density, $\mathrm{Q}_{\mathrm{cw}}$ the cold seawater flow rate, measured by FE-20, whereas the second-stage seawater distribution ratio $R_{2} t$ is $\frac{\left(T_{C W 1}-T_{C W O}\right)}{\left(T_{C W 1}-T_{C W 2}\right)}$, the latter quantity involves the measurement of seawater DCC outlet temperatures $\mathrm{T}_{\mathrm{CW} 1}$ (first stage), $\mathrm{T}_{\mathrm{CW} 2}=\mathrm{T}_{\text {out }}$ (second stage) and 


\section{Appendix B}

$\mathrm{T}_{\text {cwo }}$ (overall) by, respectively, TE-30, TE-31 and TE-22. It can be verified that $\mathrm{m}_{\mathrm{nc} 1}$ has a baseline value of $19.86 \mathrm{~g} / \mathrm{s}$.

$\mathrm{m}_{\text {steam }}$ represents what has not been condensed through the first DCC stage, that is $\chi \mathrm{m}_{\mathrm{s}}\left(1-\mathrm{F}_{1}\right)$; the total DCC inlet steam mass flow rate $\chi \mathrm{m}_{\mathrm{s}}$ may be treated as a pseudomeasurement; moreover, we have:

$$
\chi \mathrm{m}_{\mathbf{S}} \mathrm{F}_{1}=\frac{\mathrm{C}_{\mathrm{p} \rho \mathrm{cw}} \mathrm{Q}_{\mathrm{cw}}\left(\mathrm{T}_{\mathrm{cw} 1}-\mathrm{T}_{\mathrm{in}}\right)\left(\mathrm{T}_{\mathrm{CwO}}-\mathrm{T}_{\text {out }}\right)}{\mathrm{h}_{\mathrm{fg}}\left(\mathrm{T}_{\mathrm{CW} 1}-\mathrm{T}_{\text {out }}\right)}
$$

where the only additional temperature measurement is that of the cold seawater inlet, $\mathrm{T}_{\mathrm{in}}$, by TE-20.

For the second stage, the baseline values of psteam and $(\mathrm{p}-\Delta \mathrm{p})$ are, respectively, $1328 \mathrm{~Pa}$ and $1353 \mathrm{~Pa}$.

Baseline values of the main terms in the thermal effectiveness formula are given as:

$\begin{array}{llllll} & \text { Tsat } & \text { Tin } & \text { Tout } & \Delta \text { TDCC } & \text { EDCC } \\ \text { First-stage } & 11.8^{\circ} \mathrm{C} & 6.1^{\circ} \mathrm{C} & 11.4^{\circ} \mathrm{C} & & 0.89 \\ \text { Second-stage } & 11.1^{\circ} \mathrm{C} & 6.1^{\circ} \mathrm{C} & 10.5^{\circ} \mathrm{C} & & 0.83 \\ \text { Overall } & 11.8^{\circ} \mathrm{C} & & 11.2^{\circ} \mathrm{C} & 5.1^{\circ} \mathrm{C} & 0.85\end{array}$

For the first stage and overall DCC, the relevant sensitivity coefficients are obtained readily:

$\frac{\partial \varepsilon}{\partial T_{\text {in }}}=\frac{T_{\text {out }}-\left\{T_{\text {sat }}\left(p_{\text {steam }}\right)+0.28\right\}}{\left(\left\{T_{\text {sat }}\left(p_{\text {steam }}\right)+0.28\right\}-T_{\text {in }}\right)^{2}}=-\frac{\varepsilon-\varepsilon^{2}}{T_{\text {out }}-T_{\text {in }}}$,

$\frac{\partial \varepsilon}{\partial T_{\text {out }}}=\frac{\varepsilon}{T_{\text {out }}-T_{\text {in }}}$ for the first stage,

$\frac{\partial \varepsilon}{\partial T_{\text {out }}}=\frac{\varepsilon^{2}}{T_{\text {out }}-T_{\text {in }}}$ for the overall DCC,

$\frac{\partial \varepsilon}{\partial p_{\text {inlet }}}=\frac{\partial \varepsilon}{\partial T_{\text {sat }}} \frac{\partial \mathrm{T}_{\text {sat }}}{\partial p_{\text {inlet }}}=-\frac{\varepsilon^{2}}{T_{\text {out }}-\mathrm{T}_{\text {in }}} \frac{\partial \mathrm{T}_{\text {sat }}}{\partial p_{\text {inlet }}}$,

with $\frac{\partial \mathrm{T}_{\text {sat }}}{\partial \mathrm{p}_{\text {inlet }}}(1383 \mathrm{~Pa})=0.01094 \mathrm{~K} / \mathrm{Pa}$,

$\frac{\partial \varepsilon}{\partial \Delta T_{D C C}}=\frac{\left\{T_{\text {sat }}\left(p_{\text {inlet }}\right)+0.28\right\}-T_{\text {out }}}{\left(\left\{T_{\text {sat }}\left(\text { pinlet }_{\text {in }}\right)+0.28\right\}-T_{\text {in }}\right)^{2}}=\frac{\varepsilon-\varepsilon 2}{T_{\text {out }}-T_{\text {in }}}$. 
Numerical values of these coefficients are presented below as well as the resulting uncertainty on the DCC effectiveness based on a temperature measurement accuracy of $0.05^{\circ} \mathrm{C}\left(\mathrm{TE}-20\right.$ and TE-30), $\delta p_{\text {inlet }}=3 \mathrm{~Pa}(\mathrm{PT}-33)$ and $\delta \Delta \mathrm{TDCC}=0.02^{\circ} \mathrm{C}(\mathrm{TdT}-21)$ :

$$
\frac{\partial \varepsilon}{\partial T_{\text {in }}} \quad \frac{\partial \varepsilon}{\partial \Delta \mathrm{T}_{\mathrm{DCC}}} \quad \frac{\partial \varepsilon}{\partial \mathrm{T}_{\text {out }}} \quad \frac{\partial \varepsilon}{\partial \text { pinlet }^{2}} \quad \delta \varepsilon \quad \frac{\delta \varepsilon}{\varepsilon}
$$
First-stage
$-0.019$
0.168
$1.635 \times 10^{-3}$
$9.8 \times 10^{-3}$
$1.1 \%$

Overall

$$
0.025
$$

$1.553 \times 10^{-3}$

$8.5 \times 10^{-3} 1 \%$

As has been discussed, the analysis for the second stage is complicated by the cumbersome determination of the inlet partial pressure psteam. The following sensitivity coefficients have to be considered:

$$
\begin{aligned}
& \frac{\partial \varepsilon}{\partial \mathrm{T}_{\mathrm{in}}}=-\frac{\varepsilon-\varepsilon^{2}}{\mathrm{~T}_{\text {out }}-\mathrm{T}_{\mathrm{in}}}-\frac{\varepsilon^{2}}{\mathrm{~T}_{\text {out }}-\mathrm{T}_{\text {in }}} \frac{\partial \mathrm{T}_{\text {sat }}}{\partial \text { psteam }_{\text {steam }}} \frac{\partial \mathrm{p}_{\text {steam }}}{\partial \mathrm{T}_{\mathrm{in}}}=1.503 \times 10^{-3} \mathrm{~K}^{-1} \text {, } \\
& \frac{\partial \varepsilon}{\partial \mathrm{T}_{\text {out }}}=\frac{\varepsilon}{\mathrm{T}_{\text {out }}-\mathrm{T}_{\text {in }}}-\frac{\varepsilon^{2}}{\mathrm{~T}_{\text {out }}-\mathrm{T}_{\text {in }}} \frac{\partial \mathrm{T}_{\text {sat }}}{\partial \mathrm{p}_{\text {steam }}} \frac{\partial \mathrm{p}_{\text {steam }}}{\partial \mathrm{T}_{\text {out }}}=0.24935 \mathrm{~K}^{-1} \text {, } \\
& \frac{\partial \varepsilon}{\partial \mathrm{T}_{\mathrm{cw} 1}}=-\frac{\varepsilon^{2}}{\mathrm{~T}_{\text {out }}-\mathrm{T}_{\text {in }}} \frac{\partial \mathrm{T}_{\mathrm{sat}}}{\partial \mathrm{p}_{\text {steam }}} \frac{\partial \mathrm{p}_{\text {steam }}}{\partial \mathrm{T}_{\mathrm{cw} 1}}=0.17787 \mathrm{~K}^{-1} \\
& \frac{\partial \varepsilon}{\partial \mathrm{T}_{\text {cwo }}}=-\frac{\varepsilon^{2}}{\mathrm{~T}_{\text {out }}-\mathrm{T}_{\text {in }}} \frac{\partial \mathrm{T}_{\text {sat }}}{\partial \mathrm{p}_{\text {steam }}} \frac{\partial \mathrm{p}_{\text {steam }}}{\partial \mathrm{T}_{\mathrm{CwO}}}=-0.27154 \mathrm{~K}^{-1} \text {, } \\
& \frac{\partial \varepsilon}{\partial \mathrm{p}}=-\frac{\varepsilon^{2}}{\mathrm{~T}_{\text {out }}-\mathrm{T}_{\text {in }}} \frac{\partial \mathrm{T}_{\text {sat }}}{\partial \mathrm{p}_{\text {steam }}} \frac{\partial \mathrm{p}_{\text {steam }}}{\partial \mathrm{p}}=-1.743 \times 10^{-3} \mathrm{~Pa}^{-1} \\
& \frac{\partial \varepsilon}{\partial \Delta \mathrm{p}}=-\frac{\varepsilon^{2}}{\mathrm{~T}_{\text {out }}-\mathrm{T}_{\text {in }}} \frac{\partial \mathrm{T}_{\text {sat }}}{\partial \mathrm{p}_{\text {steam }}} \frac{\partial \mathrm{p}_{\text {steam }}}{\partial \Delta \mathrm{p}}=1.743 \times 10^{-3} \mathrm{~Pa}^{-1} \text {, } \\
& \frac{\partial \varepsilon}{\partial \mathrm{m}_{\mathrm{nc}}}=-\frac{\varepsilon^{2}}{\mathrm{~T}_{\text {out }}-\mathrm{T}_{\text {in }}} \frac{\partial \mathrm{T}_{\text {sat }}}{\partial \mathrm{p}_{\text {steam }}} \frac{\partial \mathrm{p}_{\text {steam }}}{\partial \mathrm{m}_{\mathrm{nc}}}=-2.1572 \times 10^{-3}(\mathrm{~g} / \mathrm{s})^{-1}, \\
& \frac{\partial \varepsilon}{\partial \mathrm{Q}_{\mathrm{cw}}}=-\frac{\varepsilon^{2}}{\mathrm{~T}_{\text {out }}-\mathrm{T}_{\mathrm{in}}} \frac{\partial \mathrm{T}_{\mathrm{sat}}}{\partial \mathrm{p}_{\text {steam }}} \frac{\partial \mathrm{p}_{\text {steam }}}{\partial \mathrm{Q}_{\mathrm{cw}}}=-0.4376\left(\mathrm{~m}^{3} / \mathrm{s}\right)^{-1} \text {, } \\
& \frac{\partial \varepsilon}{\partial \chi \mathrm{m}_{\mathrm{s}}}=-\frac{\varepsilon^{2}}{\mathrm{~T}_{\text {out }}-\mathrm{T}_{\text {in }}} \frac{\partial \mathrm{T}_{\text {sat }}}{\partial \mathrm{p}_{\text {steam }}} \frac{\partial \mathrm{p}_{\text {steam }}}{\partial \chi \mathrm{m}_{\mathrm{s}}}=0.06434(\mathrm{~kg} / \mathrm{s})^{-1}
\end{aligned}
$$

The numerical values above were obtained with $\frac{\partial T s a t}{\partial \text { psteam }}(1328.4)=0.01134 \mathrm{~K} / \mathrm{Pa}$.

Details of the straightforward, though tedious, algebraic steps required for the calculation of the various partial derivatives of psteam are left out. With an 


\section{Appendix B}

uncertainty of $0.05^{\circ} \mathrm{C}$ on all temperature readings, $\delta \mathrm{m}_{\mathrm{nc}}=1.6 \mathrm{~g} / \mathrm{s}, \delta \mathrm{p}=3 \mathrm{~Pa}$, $\delta \Delta \mathrm{p}=2.4 \mathrm{~Pa}, \delta \mathrm{Q}_{\mathrm{cW}}=0.0061 \mathrm{~m}^{3} / \mathrm{s}$ and $\delta \chi \mathrm{m}_{\mathrm{s}} \approx \chi \delta \mathrm{m}_{\mathrm{s}}=5.55 \times 10^{-2} \mathrm{~kg} / \mathrm{s}\left(\delta \mathrm{m}_{\mathrm{s}}\right.$ is evaluated elsewhere), the uncertainty for the effectiveness of the second DCC stage is $\delta \varepsilon=0.0223$, i.e. $\frac{\partial \varepsilon}{\varepsilon}=2.7 \%$.

\section{Steam Mass Flow Rate}

The steam mass flow rate, in $\mathrm{kg} / \mathrm{s}$, is estimated from the formula:

$$
\mathrm{m}_{\mathrm{S}}=\frac{\rho_{\mathrm{w}} \mathrm{c}_{\mathrm{p}} \mathrm{Q}_{\mathrm{ww}} \Delta \mathrm{T}_{\mathrm{ev}}}{\mathrm{h}_{\mathrm{fg}}}
$$

The warm seawater volume flow rate $Q_{w w}$ is measured directly, as well as the temperature difference $\Delta \mathrm{T}_{\mathrm{ev}}$ across the evaporator. The other parameters, however, are defined from appropriate curve fits functions of $T_{\text {avg }}=\frac{T_{i n}-T_{\text {out }}}{2}$ and $T_{\text {sat }}\left(p_{e v}\right)$, both expressed in ${ }^{\circ} \mathrm{C}$. More explicitly:

$$
\begin{aligned}
& \rho_{W}=1027.7154-0.04992 \mathrm{~T}_{\text {avg }}-0.006773 \mathrm{~T}_{\text {avg }^{2}}+4.766 \times 10^{-5} \mathrm{~T}_{\text {avg }^{3}} \\
& c_{p}=3.9867+4.773 \times 10^{-4} \mathrm{~T}_{\text {avg }} \\
& h_{f g}=2501.6+\left(1.86-c_{p}\right)\left\{T_{\text {sat }}\left(p_{\text {ev }}\right)+0.31\right\}
\end{aligned}
$$

With $\mathrm{T}_{\text {in }}=26^{\circ} \mathrm{C}$ and $\mathrm{T}_{\text {sat }}(2620)=21.85^{\circ} \mathrm{C}$, the baseline values of the three parameters above are, respectively, $1023.20 \mathrm{~kg} / \mathrm{m}^{3}, 3.998 \mathrm{~kJ} / \mathrm{kg}-\mathrm{K}$ and $2454.2 \mathrm{~kJ} / \mathrm{kg}$. With $\mathrm{Q}_{\mathrm{ww}}=0.6057 \mathrm{~m}^{3} / \mathrm{s}$ and $\Delta \mathrm{T}_{\mathrm{ev}}=3.5^{\circ} \mathrm{C}$, the nominal steam flow rate is $3.536 \mathrm{~kg} / \mathrm{s}$.

The following sensitivity coefficients need to be considered:

$$
\begin{aligned}
& \frac{\partial \mathrm{m}_{\mathrm{s}}}{\partial \mathrm{Q}_{\mathrm{ww}}}=\frac{\rho_{w} \mathrm{c}_{\mathrm{p}} \Delta \mathrm{T}_{\mathrm{ev}}}{\mathrm{h} f \mathrm{fg}}=\frac{\mathrm{m}_{\mathrm{s}}}{\mathrm{Q}_{\mathrm{ww}}} \approx 5.8377 \mathrm{~kg} / \mathrm{m}^{3} \\
& \frac{\partial \mathrm{m}_{\mathrm{S}}}{\partial \Delta \mathrm{T}_{\mathrm{eV}}}=\frac{\rho_{\mathrm{w}} \mathrm{C}_{\mathrm{p}} \mathrm{Q}_{\mathrm{ww}}}{\mathrm{h}_{\mathrm{fg}}}=\frac{\mathrm{m}_{\mathrm{S}}}{\Delta \mathrm{T}_{\mathrm{eV}}} \approx 1.0114 \mathrm{~kg} / \mathrm{s}-\mathrm{K} \\
& \frac{\partial \mathrm{m}_{\mathrm{s}}}{\partial \mathrm{pev}_{\mathrm{e}}}=\frac{\partial \mathrm{m}_{\mathrm{s}}}{\partial \mathrm{h}_{\mathrm{fg}}} \frac{\partial \mathrm{h}_{\mathrm{fg}}}{\partial \mathrm{T}_{\text {sat }}} \frac{\partial \mathrm{T}_{\text {sat }}}{\partial \mathrm{p}_{\mathrm{ev}}}=-\frac{\left(1.86-\mathrm{c}_{\mathrm{p}}\right) \mathrm{m}_{\mathrm{s}}}{\mathrm{h}_{\mathrm{fg}}} \frac{\partial \mathrm{T}_{\text {sat }}}{\partial \mathrm{pev}_{\mathrm{ev}}} \approx 1.93 \times 10^{-5} \mathrm{~kg} / \mathrm{s}-\mathrm{Pa} \\
& \frac{\partial \mathrm{m}_{S}}{\partial \mathrm{T}_{\text {in }}}=\frac{\partial \mathrm{m}_{\mathrm{S}}}{\partial \mathrm{T}_{\text {out }}}=0.5 \frac{\partial \mathrm{m}_{S}}{\partial \mathrm{T}_{\text {avg }}} \approx-0.0005 \mathrm{~kg} / \mathrm{s}-\mathrm{K}
\end{aligned}
$$

with $\frac{\partial \mathrm{m}_{s}}{\partial T_{\text {avg }}}=\frac{\partial \mathrm{m}_{s}}{\partial \rho_{\mathrm{W}}} \frac{\partial \rho_{\mathrm{W}}}{\partial \mathrm{T}_{\mathrm{avg}}}+\frac{\partial \mathrm{m}_{s}}{\partial c_{\mathrm{p}}} \frac{\partial c_{p}}{\partial T_{\text {avg }}}$ 
Appendix B

$$
=\frac{m_{s}}{\rho_{w}} \frac{\partial \rho_{w}}{\partial T_{\text {avg }}}+\frac{h_{f g}+c_{p}\left\{T_{\text {sat }}\left(p_{e v}\right)+0.31\right\}}{h_{f g}{ }^{2}} \frac{\partial c_{p}}{\partial T_{\text {avg }}}
$$

With $\delta Q_{w w}=0.0091 \mathrm{~m}^{3} / \mathrm{s}(\mathrm{FE}-10), \delta \Delta \mathrm{T}_{\mathrm{ev}}=0.02^{\circ} \mathrm{C}(\mathrm{TdT}-11), \delta \mathrm{pev}=5.4 \mathrm{~Pa}(\mathrm{PT}-30)$, $\delta \mathrm{T}_{\text {in }}=\delta \mathrm{T}_{\mathrm{out}}=0.05^{\circ} \mathrm{C}(\mathrm{TE}-10$ and $\mathrm{TE}-11)$, and the above sensitivity coefficients, it is obvious that the contributions of $\mathrm{T}_{\mathrm{in}}, \mathrm{T}_{\mathrm{out}}$ and $\mathrm{p}_{\mathrm{ev}}$ to $\delta \mathrm{m}_{\mathrm{s}}$ are negligible. Consequently:

$$
\delta \mathrm{m}_{\mathrm{s}} \approx \sqrt{\left(\frac{\partial \mathrm{m}_{\mathrm{s}}}{\partial \mathrm{Q}_{\mathrm{ww}}} \delta \mathrm{Q}_{\mathrm{ww}}\right)^{2}+\left(\frac{\partial \mathrm{m}_{\mathrm{s}}}{\partial \Delta \mathrm{T}_{\mathrm{ev}}} \delta \Delta \mathrm{T}_{\mathrm{ev}}\right)^{2}}=0.057 \mathrm{~kg} / \mathrm{s}
$$

i.e. $\quad \frac{\delta m_{S}}{m_{S}} \approx 1.6 \%$

It is important here to perform a direct differential temperature measurement of $\Delta \mathrm{T}_{\text {ev }}$ instead of taking the difference between $\mathrm{T}_{\mathrm{in}}$ and $\mathrm{T}_{\text {out }}$. Not only the differential device is more sensitive, but the dominant sensitivity factor in the above formula, which is related to $\Delta \mathrm{T}_{\mathrm{ev}}$, would appear twice if the absolute temperatures were used: the overall effect would approximately double $\frac{\delta \mathrm{m}_{\mathrm{s}}}{\mathrm{m}_{\mathrm{s}}}$.

\section{Isentropic Power across the Turbine}

The isentropic power across the turbine, all the way to the diffuser exit, is defined as $P_{i s e}=m_{s} \Delta h_{i s e}$. Pise is an abstraction which is used in evaluating the performance of the turbine, via its efficiency.

While information on the steam mass flow rate was provided above, the nominal value of the isentropic enthalpy drop $\Delta \mathrm{h}_{\mathrm{ise}}$ is $83.84 \mathrm{~kJ} / \mathrm{kg}$, corresponding to turbine inlet and outlet pressures of 2611 and $1383 \mathrm{~Pa}$, respectively, and thermodynamic properties linearly interpolated from the ASME Steam Tables (1977). Thus, the baseline value of Pise is $296.45 \mathrm{~kW}$. More specifically, we have:

$$
\Delta h_{\text {ise }}=h_{1}-\left\{h_{f 2}+\frac{s 1-s f 2}{s_{f g} 2} h_{f g}\right\}
$$

In the above formula, subscripts 1 and 2 define the turbine inlet (saturated steam) and the diffuser outlet (two-phase mixture), respectively. The isentropic nature of $\Delta h_{i s e}$ is reflected in the presence of $s_{1}$ (instead of the real, unknown $s_{2}$ ) in the steam quality $\chi$ ise $=\frac{s 1-s f 2}{s f g 2}$

State 1 variables depend upon the measurement of the turbine inlet pressure $\mathrm{p}_{1}$, while state 2 variables are functions of the turbine outlet pressure $\mathrm{p}_{2}$. As mentioned above, all components of $\Delta$ hise are read, for example, from the ASME Steam Tables (1977), with the appropriate pressure as an entry. Thus, we have:
$\mathrm{h}_{1}$
$(\mathrm{~kJ} / \mathrm{kg})$
$\stackrel{\text { s1 }}{\text { (kJ/kg-K) }}$
sfg2
$(\mathrm{kJ} / \mathrm{kg}-\mathrm{K})$
sf2
$(\mathrm{kJ} / \mathrm{kg}-\mathrm{K})$
hfg2
(kJ/kg)
hf2
2541.43
8.628
8.681
0.1775
2473.66
(kJ/kg)
49.51 


\section{Appendix B}

Neglecting the influences of $p_{e v}, T_{i n}$ and $T_{\text {out }}$ upon $m_{S}$, as they proved to be negligible, the two measured quantities affecting $P_{i s e}$ through $\mathrm{m}_{\mathrm{S}}$ are $\mathrm{Q}_{\mathrm{ww}}$ and $\Delta \mathrm{T}_{\mathrm{ev}}$ :

$$
\begin{aligned}
& \frac{\partial \mathrm{P}_{\text {ise }}}{\partial \Delta \mathrm{T}_{\mathrm{ev}}}=\frac{\partial \mathrm{P}_{\text {ise }}}{\partial \mathrm{m}_{\mathrm{s}}} \frac{\partial \mathrm{m}_{\mathrm{s}}}{\partial \Delta \mathrm{T}_{\mathrm{ev}}}=\Delta \mathrm{h}_{\text {ise }} \frac{\partial \mathrm{m}_{\mathrm{s}}}{\partial \Delta \mathrm{T}_{\mathrm{ev}}} \approx 84.796 \mathrm{~kW} / \mathrm{K} \\
& \frac{\partial \mathrm{P}_{\mathrm{ise}}}{\partial \mathrm{Q}_{\mathrm{ww}}}=\frac{\partial \mathrm{P}_{\text {ise }}}{\partial \mathrm{m}_{\mathrm{s}}} \frac{\partial \mathrm{m}_{\mathrm{S}}}{\partial \mathrm{Q}_{\mathrm{ww}}}=\Delta \mathrm{h}_{\text {ise }} \frac{\partial \mathrm{m}_{\mathrm{S}}}{\partial \mathrm{Q}_{\mathrm{ww}}} \approx 489.43 \mathrm{~kJ} / \mathrm{m}^{3}
\end{aligned}
$$

Moreover, the other two measured parameters used to determine $\Delta \mathrm{h}_{\mathrm{ise}}$ are $\mathrm{p}_{1}$ and $\mathrm{p}_{2}$. From the ASME Steam Tables (1988), we obtain (in SI units):

$$
\begin{array}{cccccc}
\frac{\partial \mathrm{h}_{1}}{\partial \mathrm{p} 1} & \frac{\partial \mathrm{s} 1}{\partial \mathrm{p} 1} & \frac{\partial \mathrm{sf} 2}{\partial \mathrm{p} 2} & \frac{\partial \mathrm{sfg} 2}{\partial \mathrm{p} 2} & \frac{\partial \mathrm{h}_{2}}{\partial \mathrm{p} 2} & \frac{\partial \mathrm{hfg}_{\mathrm{f}}}{\partial \mathrm{p} 2} \\
1.15 \times 10^{-2} & -1.33 \times 10^{-4} & 1.65 \times 10^{-4} & -4.34 \times 10^{-4} & 4.69 \times 10^{-2} & -2.70 \times 10^{-2}
\end{array}
$$

(the above values are linear slope coefficients between 2600 and $2800 \mathrm{~Pa}$ for subscript 1 parameters, and between 1300 and $1400 \mathrm{~Pa}$ for subscript 2 parameters)

On the other hand, we have:

$\frac{\partial \mathrm{P}_{\text {ise }}}{\partial \mathrm{p}_{1}}=\frac{\partial \mathrm{P}_{\text {ise }}}{\partial \Delta \mathrm{h}_{\text {ise }}} \frac{\partial \Delta \mathrm{h}_{\text {ise }}}{\partial \mathrm{p}_{1}}=\mathrm{m}_{\mathrm{s}}\left\{\frac{\partial \mathrm{h}_{1}}{\partial \mathrm{p}_{1}}-\frac{\mathrm{h}_{\mathrm{fg} 2}}{\mathrm{sfg} 2} \frac{\partial \mathrm{s}_{1}}{\partial \mathrm{p}_{1}}\right\} \approx 0.1747 \mathrm{~kW} / \mathrm{Pa}$

$\frac{\partial \mathrm{P}_{\text {ise }}}{\partial \mathrm{p} 2}=\frac{\partial \mathrm{P}_{\text {ise }}}{\partial \Delta \mathrm{h}_{\text {ise }}} \frac{\partial \Delta \mathrm{h}_{\text {ise }}}{\partial \mathrm{p}_{2}}$

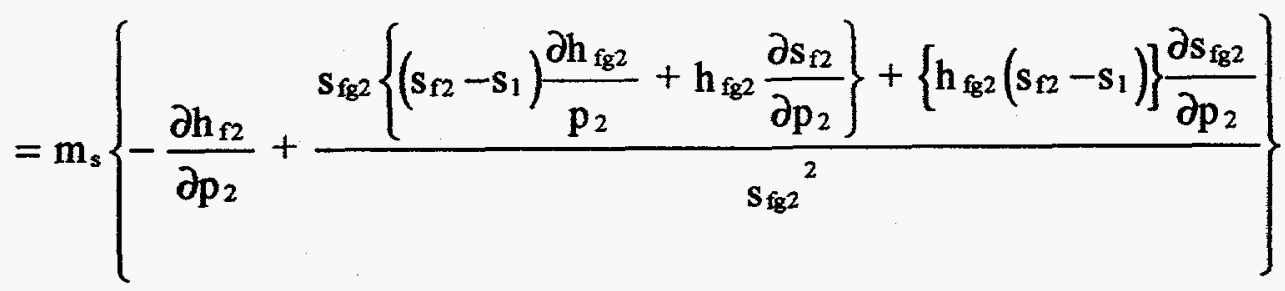

$\approx-0.3323 \mathrm{~kW} / \mathrm{Pa}$

With $\delta Q_{\mathrm{ww}}=0.0091 \mathrm{~m}^{3} / \mathrm{s}(\mathrm{FE}-10), \delta \Delta \mathrm{T}_{\mathrm{ev}}=0.02^{\circ} \mathrm{C}(\mathrm{TdT}-11), \delta \mathrm{p}_{1}=5.4 \mathrm{~Pa}(\mathrm{PT}-32)$, $\delta \mathrm{p}_{2}=3 \mathrm{~Pa}(\mathrm{PT}-33)$, and the above sensitivity coefficients, we can write:

$$
\delta P_{\text {ise }}=\sqrt{\left(\frac{\partial P_{\text {ise }}}{\partial Q_{\text {ww }}} \delta Q_{\text {ww }}\right)^{2}+\left(\frac{\partial P_{\text {ise }}}{\partial \Delta T_{\text {ev }}} \delta \Delta T_{\text {ev }}\right)^{2}+\left(\frac{\partial P_{\text {ise }}}{\partial p_{1}} \delta p_{1}\right)^{2}+\left(\frac{\partial P_{\text {ise }}}{\partial p_{2}} \delta p_{2}\right)^{2}}
$$

i.e. $\quad \delta P_{i s e} \approx 4.96 \mathrm{~kW}$ and $\frac{\delta P_{\text {ise }}}{P_{\text {ise }}} \approx 1.7 \%$. 


\section{Appendix B}

\section{Overall Fraction of Steam Condensed}

The formula for the fraction of steam condensed by the direct-contact condenser (DCC) is:

$$
F=\frac{\rho_{w} c_{p} Q_{c w} \Delta T_{D C C} 1}{h_{f g}} \frac{1}{\chi m_{S}}
$$

$\mathrm{F}$ has a nominal value of 0.99 . $\mathrm{Q}_{\mathrm{cw}}$ is the volumetric flow rate of cold seawater into the DCC, and has a baseline value of $0.4031 \mathrm{~m}^{3} / \mathrm{s}$, whereas the nominal cold seawater should be evaluated at an average temperature across the DCC. This ratio, however, is formally similar to the expression for $\mathrm{m}_{s}$, illustrated earlier (we simply deal here with the DCC instead of the evaporator). It was shown that only the measurement of $\mathrm{Q}_{\mathrm{ww}}$ and $\Delta \mathrm{T}_{\mathrm{ev}}$ influenced the uncertainty of the derived parameter $\mathrm{m}_{\mathrm{s}}$. Similarly, it is clear that only $\mathrm{Q}_{C \mathrm{C}}$ and $\Delta \mathrm{T}_{\mathrm{DCC}}$ will practically affect the first ratio in the definition of $F$. Thus, we can immediately write:

$\frac{\partial F}{\partial Q_{w w}}=-\frac{F}{Q_{w w}} \approx-1.62 \mathrm{~s} / \mathrm{m}^{3}$

$\frac{\partial \mathrm{F}}{\partial \Delta \mathrm{T}_{\mathrm{ev}}}=-\frac{\mathrm{F}}{\Delta \mathrm{T}_{\mathrm{ev}}} \approx-0.281 \mathrm{~K}^{-1}$

$\frac{\partial \mathrm{F}}{\partial \mathrm{Q}_{\mathrm{CW}}}=\frac{\mathrm{F}}{\mathrm{Q}_{\mathrm{cW}}} \approx 2.46 \mathrm{~s} / \mathrm{m}^{3}$

$\frac{\partial \mathrm{F}}{\partial \Delta \mathrm{T}_{\mathrm{DCC}}}=\frac{\mathrm{F}}{\Delta \mathrm{T}_{\mathrm{DCC}}} \approx 0.186 \mathrm{~K}^{-1}$

On the other hand, $\chi$ is a function of turbine inlet and outlet pressures:

$$
\chi=\frac{s_{2}-s_{f 2}}{s f g 2} .
$$

Since the entropy $s 2$ is unknown, $\chi$ is estimated by means of the baseline turbine efficiency $\eta_{t}$, defined as:

$$
\eta_{t}=\frac{h_{1}-\left\{h_{f 2}+\chi h_{f g}\right\}}{h_{1}-\left\{h_{f 2}+\frac{s 1-s_{2}}{s_{f g} 2} h_{f g}\right\}}
$$

Thus, $\chi=\frac{h_{1}-h_{f 2}}{h_{f g}}\left(1-\eta_{t}\right)+\eta_{t} \frac{s 1-s f 2}{s_{f g} 2}$

Baseline values for $\chi$ and $\eta_{t}$ are 0.98 and 0.84 , respectively. Evaluating the sensitivity of $\mathrm{F}$ to $\mathrm{p}_{1}$ and $\mathrm{p}_{2}$, via $\chi$, is not easy because $\eta_{\mathrm{t}}$ also depends upon these pressures in a not straightforward way. 'Pretending', however, that $\chi$ can be replaced by $\chi$ ise, in estimating the partial derivatives of steam quality upon turbine inlet and outlet 
pressures, shows that the order of magnitude of $\frac{\partial \mathrm{F}}{\partial \mathrm{p}_{1}}$ and $\frac{\partial \mathrm{F}}{\partial \mathrm{p} 2}$ is probably too small, of the order of $10^{-5} \mathrm{~Pa}^{-1}$, to affect the uncertainty on $\mathrm{F}$. Therefore, with previously used instrument uncertainties and $8 \mathrm{Q}_{\mathrm{cw}}=0.0061 \mathrm{~m}^{3} / \mathrm{s}(\mathrm{FE}-20)$ :

$$
\frac{\delta F}{F}=\sqrt{\left(\frac{\partial F}{\partial Q_{w w}} \delta Q_{w w}\right)^{2}+\left(\frac{\partial F}{\partial \Delta T_{e v}} \delta T_{e v}\right)^{2}+\left(\frac{\partial F}{\partial Q_{c w}} \delta Q_{c w}\right)^{2}+\left(\frac{\partial F}{\partial T_{D C C}} \delta T_{D C C}\right)^{2}}
$$

i.e. $\frac{\delta \mathrm{F}}{\mathrm{F}} \approx 2.2 \%$

\section{References}

ASME Steam Tables in SI (Metric) Units for Instructional Use, ASME, New York,1977.

Benedict, R. P., Fundamentals of Temperature, Pressure and Flow Measurements, John Wiley \& Sons, New York, 1984.

Miller, R. W., Elow Measurement Engineering Handbook, McGraw-Hill, New York, 1983.

Oney, S. K., and H. J. Krock, "Noncondensable gas composition and outgassing rates in the open-cycle OTEC system with direct-contact condensation", Proc. Int. Conf. on Ocean Energy Recovery, ASCE Pub., 1989. 